\title{
Biomarkers for prediction and targeted prevention of Alzheimer's and Parkinson's diseases: evaluation of drug clinical efficacy
}

\author{
Silvia A. Mandel • Micaela Morelli • Ilan Halperin • \\ Amos D. Korczyn
}

Received: 10 May 2010 / Accepted: 5 June 2010 / Published online: 29 June 2010

(C) European Association for Predictive, Preventive and Personalised Medicine 2010

\begin{abstract}
Neurodegenerative diseases like Parkinson's disease (PD) and Alzheimer's disease (AD) are considered disorders of multifactorial origin, inevitably progressive and having a long preclinical period. Therefore, the availability of biological markers or biomarkers (BMs) for early disease diagnosis will impact the management of $\mathrm{AD}$ and $\mathrm{PD}$ in several dimensions; it will 1) help to capture high-risk individuals before symptoms develop, a stage where prevention efforts might be expected to have their greatest impact; 2) provide a measure of disease progression that can be evaluated objectively, while clinical measures are much less accurate; 3) help to discriminate between true $\mathrm{AD}$ or PD and other causes of a similar clinical syndrome; 4) delineate pathophysiological processes responsible for the disease; 5) determine the clinical efficacy of novel, disease-modifying (neuroprotective) strategies. In the long run the availability of reliable BMs will significantly advance the research and therapeutics of $\mathrm{AD}$ and $\mathrm{PD}$.
\end{abstract}

\author{
S. A. Mandel $(\bowtie)$ \\ Eve Topf Center for Neurodegenerative Diseases \\ Research and Department of Molecular Pharmacology, \\ Faculty of Medicine, Technion, \\ Haifa, Israel \\ e-mail:mandel@tx.technion.ac.il

\section{Morelli} \\ Department of Toxicology and Centre of Excellence \\ for Neurobiology of Dependence, University of Cagliari, \\ Cagliari, Italy

\section{Halperin} \\ The Israeli Psycological Association, \\ Beersheba, Israel \\ A. D. Korczyn \\ Tel-Aviv University Medical School Sieratzki Chair of Neurology, \\ Ramat-Aviv, Israel
}

Keywords Alzheimer's disease - Apolipoprotein E · Biomarker - Disease modifying therapy $\cdot$ Parkinson's disease $\cdot$ Neuroimaging

\section{Introduction}

Parkinson's disease (PD) and Alzheimer's disease (AD) are neurodegenerative disorders sharing in common a number of characteristics; the more common sporadic forms are considered to be multifactorial in origin, inevitably progressive and have a long preclinical period. Currently, the clinical diagnosis of PD can be made when motor symptoms occur, though the disease has originated several years earlier. Furthermore, at its initial stages PD may be confounded by other diseases, such as essential tremor, multiple system atrophy (MSA) and progressive supranuclear palsy (PSP) $[1,2]$. Similar to PD, the pathological process characteristic of $\mathrm{AD}$ begins decades before the first symptoms of cognitive dysfunctions, thus making it difficult to reliably identify pathology based on the clinical phenotype alone. AD may share clinical features with many other dementing disorders including vascular dementia $(\mathrm{VaD})$, dementia with Lewy bodies (DLB), PSP and others $[3,4]$. Therefore, the availability of biological markers or biomarkers (BM) for early disease diagnosis will impact the management of $\mathrm{AD}$ and $\mathrm{PD}$ in several dimensions: first, it will allow to capture high-risk individuals before symptoms develop; second, it will help to discriminate between true $\mathrm{AD}$ or PD and other causes of a similar clinical syndrome. In addition, a BM may help to determine the clinical efficacy of new neuroprotective therapies. The development of neuroprotective therapies is greatly hindered by the lack of markers capable of serving as surrogate objective endpoints for clinical trials testing these treatments. 
Among the diverse, significant challenges facing the clinicians, is the improvement of diagnostic measures in order to 1) detect early/subtle symptoms, a phase in which prevention efforts might be expected to have their greatest impact; 2) provide a measure of disease progression that can be evaluated objectively, while clinical measure are much less accurate; 3) provide evidence for relevant biological activity of an experimental drug; 4) delineate pathophysiological processes responsible for the disease. Regrettably, clinical endpoints such as changes in patient's features, or the time it takes to reach specific diseaseassociated disabilities may vary drastically among patients or clinical raters and require long trial durations and a large number of subjects before significant effects are seen, thus being inaccurate and expensive measures of disease progression. Also, the current assessment of clinical disease improvement does not always allow for a clear distinction between symptom-masking versus disease-modifying (neuroprotective) therapies.

BMs have been defined as "cellular, biochemical or molecular alterations that are measurable in the biological media such as human tissues, cells or fluids" [5]. Others broaden the definition to include biological characteristics that can be measured objectively and evaluated as indicators of normal biological processes, or pharmacological responses to therapeutic intervention [6], such as electrophysiological activities (EEG) or responses (e.g. evoked reactions). BMs for detection of neurodegenerative disorders can be divided into three main categories: genetic, neuroimaging, and biochemical. Normally, neuroimaging and biochemical BMs mark the presence of pathology, while genetic markers can only serve for risk assessment.

Each BM must be evaluated for its specificity and sensitivity for a given disease. The same applies for BMs that are used to define stages of disease severity [7]. However, increased specificity is usually achieved at the expense of reduced sensitivity and vice versa. Therefore a compromise must be achieved. Whether one attempts to achieve maximal sensitivity depends on the aims of the study and the 'price' which is involved. For example, if a cheap, safe and effective therapy is available for a condition, a less specific test can be used even if this will eventually result in treatment being given to people who do not have the disease. Conversely, if the treatment is costly or has significant adverse effects, a more specific test will be required.

This review underlines the utility of genetic, neuroimaging, and biochemical BMs in the clinic, focusing on their predicitive diagnostic value for targeted prevention of $\mathrm{AD}$ and $\mathrm{PD}$ and their potential use to monitor disease progression and its modification by therapy, as well as to delineate the pathobiological (e.g. molecular) processes underlying the diseases.

\section{Biomarkers for Alzheimer's disease}

During the last century the population has shown a staggering increase in the proportion of elderly members. All epidemiological studies of dementia show a strong correlation between prevalence and age. In the age group of 60 to 64 years the prevalence of dementia is below $1 \%$; it then doubles approximately every 5 years reaching a prevalence of about $50 \%$ among those age 90 years or older $[8,9]$. This high frequency is important in terms of social policy because it frequently marks the need for permanent nursing care [10].

A variety of neurodegenerative pathologies are associated with the development of late life dementia (LLD), while $\mathrm{AD}$ is considered by far the most prevalent. $60-75$ percent of all cases of dementia are diagnosed as $\mathrm{AD}[8,11]$. However, the clinical diagnosis of demented patients is far from perfect. At present, diagnosis is based on clinical constellation of symptoms and manifestations. In order to reach a consensus, diagnostic criteria have been formulated, for example the Diagnostic and Statistical Manual of Mental Disorders (DSM) or the International Classification of Diseases (ICD) criteria, and more specific ones like McKhann's criteria [12].

$\mathrm{AD}$ is associated with progressive memory deterioration and worsening of additional cognitive functions. One factor responsible for cognitive deterioration is a loss of cholinergic transmission in cortical brain regions innervated by neurons arising in the nucleus basalis of Meynert. The presence of extracellular plaques containing deposits of proteins, particularly, $\beta$-amyloid, and intracellular neurofibrillary tangles (NFTs) are hallmarks of the pathology of $\mathrm{AD}$ and are thought to contribute to the cognitive deficit. These changes however, may result from destructive processes causing disruption of microtubule assembly and synaptic loss, rather than its cause. They could contribute to further neuronal damage and disease progression [3]. Another common form of dementia is that of the Lewy body type, DLB, which is characterised by the presence of intracytoplasmic, eosinophilic, neuronal inclusion bodies in the neocortex, limbic areas and subcortical nuclei. These are thought to be responsible for the neuronal damage and give rise to the cognitive deficits, extrapyramidal symptoms and behavioural abnormalities. Depressive symptoms occur in a large proportion of subjects with $\mathrm{AD}$ and DLB dementia and with PD and may occur several years before the typical cognitive and motor symptoms appear. This has been attributed to degeneration of noradrenaline and serotonin neurons innervating the limbic system.

The increasing prevalence of $\mathrm{AD}$ and the devastating consequences of LLD motivate the drive to develop diagnostic BMs to reliably identify the pathology associated with this disorder, particularly at an early 
stage of the pathologic process. It is also quite possible that BMs may also help to identify subclasses of the demented disorders [13].

BMs based on detection of pathology may diagnose the process more definitively and reliably then the traditional cognitive and neurological phenotypes. Strategies to accomplish these include for example, (1) the detection of altered levels of tau and amyloid proteins in the cerebrospinal fluid (CSF), (2) the use of structural Magnetic Resonance Imaging (MRI) to identify diseasespecific patterns of regional or generalised atrophy (3) and direct imaging of amyloid deposits using positron emission tomography (PET) and single photon emission computerised tomography (SPECT). At present, diagnostic markers are used extensively in the clinic, but mainly as exclusionary markers, for example elevated levels of 14-33 or tau protein in the CSF, characteristic EEG discharges and MRI signals which suggest the existence of prion diseases, "exclude" the diagnosis of AD [14].

There are several reasons for the emerging need to incorporate BMs into diagnostic criteria used by clinicians to indentify the pathology responsible for the patient's cognitive failure. These include: (1) the fact that a single type of pathology can produce a variety of different types of cognitive and behavioral symptoms. This clinical heterogeneity makes it difficult to reliably identify the pathology based solely on the clinical phenotype. Even more important is the recognition that a variety of neurodegenerative and vascular pathologies can coexist, and in fact it seems that most patients with LLD have mixed pathologies underlying the cognitive deterioration. (2) A particular problem is whether AD can be detected at a very early stage, much before clinical criteria sufficient to diagnose dementia, appear. This will become especially important since treatment can achieve maximum benefit when it is given in the earliest stages of the disease $[15,16]$.

\section{Conceptual issues}

One central problem is the concept of $\mathrm{AD}$ as a single disease entity [17]. However the definition of AD is based on the final result, the accumulation of $\beta$-amyloid deposits and NFTs. It is quite clear that similar, or identical, pathological lesions can results from different causes, e.g. specific genetic mutations cause the disease in a minority of subjects, while in the majority $\mathrm{AD}$ is sporadic, and presumably is the consequence of multiple environmental factors. It is thus not clear to what extent the initial biological processes are similar. This of course has important implications on the issue at hand, i.e. whether identical BM will be reliable in each aetiologic subtype.

A practical question is what are the BMs used for. In reality, a diagnostic BM which will distinguish normal people from advanced AD is not very useful. The helpful $\mathrm{BM}$ is one that is informative in situations where clinical data are not sufficient to allow a clear diagnosis [18].

BMs can therefore be used for different purposes:

1. Identify 'at risk' people prior to disease onset.

2. Identify people with early $\mathrm{AD}$, prior to the development of the full clinical phenotype, for example at the Mild Cognitive Impairment-(MCI) stage. $19-50 \%$ of all sufferers from MCI will develop to dementia over 4 years [19].

3. Differentiate AD from other neurodegenetive processes in demented individuals.

4. To monitor effects of intervention on the evolution of the disease.

5. To gain insight into processes underlying the pathological changes in brain areas known to be linked with $\mathrm{AD}$ in its various degrees of severity, i.e. temporal, parietal, and frontal lobes cortex.

As will be seen, most if not all BMs which have been suggested reveal statistical differences between groups (e.g. normals versus early $\mathrm{AD}$, or $\mathrm{AD}$ versus other LLD), but none of them has been shown to be sufficiently sensitive to allow a definite diagnosis in an individual person. This is because of the heterogeneity which exists even at baseline, for example in hippocampal size, among different individuals. However, repeated studies may show a change such that a more abnormal result (e.g. a hippocampus which is smaller than at baseline, yet still within normal limits) might be more helpful in detecting a disease process [20].

\section{Genetic markers of $\mathrm{AD}$}

Molecular genetic studies of neurodegenerative disorders have been useful both in identifying genes that may be causative or associated with specific diseases and in uncovering functional mechanisms subserved by products of those genes that may be markers for diagnosis or early detection. Genetics provide information to assess who is at risk, but except for the case of rare genetic disorders, it does not provide complete information when or whether those 'at risk' may expect the onset of neurodegeneration.

In the case of $\mathrm{AD}$, mutations in three genes are now known to cause the disorder in families with an autosomal dominant pattern of inheritance. Detection of the mutations in these genes can identify at-risk family members, but it is not generalisable to sporadic $\mathrm{AD}[21,22]$. In $\mathrm{AD}$, the identification of $\beta$-amyloid precursor protein (APP) and presenilin 1 and 2 mutations has strengthened the amyloid hypothesis and identified potential sites for pharmacological interventions.

An important relevant issue is the genetic association with the increased risk of developing $\mathrm{AD}$ in families that 
carry the apolipoprotein E $\varepsilon 4$ allele. ApoE is a protein involved in the transport of cholesterol. Apart from its presence in the plasma, it is also produced by astrocytes and neurons in the CNS to support and repair neurons. The apoE gene is localised on chromosome 19 and three alleles are described in humans $(\varepsilon 2, \varepsilon 3$ and $\varepsilon 4)$. A growing volume of evidence has reported an association of apoE $\varepsilon 4$ and late-onset familial and sporadic AD [23]. As many as $40-50 \%$ of AD patients possess $\varepsilon 4$ allele compared to $15-$ $25 \%$ of controls [24]. Subjects homozygous for $\varepsilon 4$ are reported to have 6 to 8 -fold increased risk of developing AD compared to the risk of heterozygotic subjects (increased by 2 to 4 -fold) [23]. However only $40 \%$ of all patients with LOAD have one $\mathrm{apoE} \varepsilon 4$, and to date no other LOAD gene has been conclusively proven. At present the existence of $\varepsilon 4$, and particularly $\varepsilon 4 / \varepsilon 4$ genotype in an individual merely increases the risk of developing AD [25]. Recent data, discovered using phylogenetic analysis, suggest that a variable length poly-T sequence polymorphism in the translocase of outer mitochondrial membrane 40 homolog (TOMM40) gene, adjacent to the APOE gene, is associated with age-of-onset of $\mathrm{AD}$. When linked to APOE $\varepsilon 3$, the longer TOMM40 poly-T repeats are associated with earlier age at onset and the shorter with later age at onset, questioning the alleged 'neutral' role of the $\varepsilon 3$ allele in $A D$ risk. [26]. More detailed information on potential genetic risk factors for late-onset AD (LOAD), is provided by a separate review in this journal-issue [27].

One important PET study [28] found that cognitively normal 50 to 65 years-old $\varepsilon 4$ carriers have low rate of cerebral glucose metabolism in brain regions associated with AD (posterior cingulate, parietal, temporal and prefrontal cortex). The last findings motivated the authors [29] to consider whether $\varepsilon 4$ carriers have these regional brain abnormalities even when still young. For this purpose apolipoprotein $\mathrm{E}$ genotype were established from healthy young adults (20-39 years of age). Clinical rating, neuropsychological tests and PET where preformed in 12 $\varepsilon 4$ heterozygotes, all with $\varepsilon 3 / \varepsilon 4$ genotype and 15 noncarriers of $\varepsilon 4$ allele. The results of this study indicated that even cognitively healthy $\varepsilon 4$ carriers have functional brain abnormalities in regions that are associated with $\mathrm{AD}$ several decades later. These abnormalities were detected even though the young $\varepsilon 4$ carriers were restricted to person with one copy of this allele who tends to have lower risk of $\mathrm{AD}$ than those with two $\varepsilon 4$ allele copies.

In a recent fMRI study by Bookheimer and coworkers [30] the researchers tried to determine the relationship between brain responses to tasks requiring memory and the genetic risk of AD by performing APOE genotyping and MRI of the brain in older persons with intact cognition. Both the magnitude and the extent of brain activation during memory-activation tasks in regions affected by $\mathrm{AD}$, including the left hippocampal, parietal, and prefrontal regions, were greater among the carriers of the apoE $\varepsilon 4$ allele than among the carriers of the apoE $\varepsilon 3$ allele. During periods of recall, the carriers of the apoE $\varepsilon 4$ allele had a greater average increase in signal intensity in the hippocampal region and a greater mean number of activated regions throughout the brain than did carriers of the apoE $\varepsilon 3$ allele. This suggests that the patterns of brain activation during tasks requiring memory differ depending on the genetic risk of $\mathrm{AD}$ and may predict subsequent decline in memory.

Obviously, genetic markers are by themselves not sufficient to make a clinical diagnosis of AD. The mutation, if it exists, has been there since conception and birth of the individual. Thus a person carrying a disease-causing mutation is not immune to the development of other diseases, and the diagnosis must be supported by clinical evidence.

Transgenic animal models have been constructed that mimic some aspects of the AD pathology [31] and proof of principle studies have been able to reduce the transgeneinduced pathology with strategies that use anti-inflammatory medications, antibody stimulation by immunisation, or other disruptions of pathobiological cascades [32-34]. However, the relevance of these models to human sporadic $\mathrm{AD}$ has been contested [35].

Ultimately, sensitive methods for identifying genetic background may provide a better means for characterising a statistical risk before disease manifestation. Nevertheless, it seems logical to assume that effective identification of the disease must combine genetic characteristics with identifying a clinical phenotype or/and additional BMs, either for early detection or for the more correct diagnosis of an already existing disease [36].

\section{Neuroimaging markers}

During the past decade neuroimaging has emerged as a strategy to attempt defining neurodegenerative disorders phenotype in both preclinical and early clinical disease stages. Imaging provides a window to the underlying processes in these disorders since loss of brain volume is one of the earliest consequences of neuron death. When extreme, it can distinguish from age related changes. It has long been appreciated that $\mathrm{AD}$ produces regionally specific neurodegeneration starting in entorhinal cortex and the hippocampus [37-39]. Using standardised qualitative visual rating scale, it is possible to detect these changes on MRI images [40].

Retrospective and prospective neuropathological studies have shown neuritic plaques and neurofibrillary tangles, commonly found in $\mathrm{AD}$, at approximately $60 \%$ of $\mathrm{MCI}$ patients that have come to autopsy [41]. These new data 
prove, as long suspected, that significant pathological changes occur in the presymptomatic and very early clinical symptomatic stages of $\mathrm{AD}$ and have further encouraged efforts to find clinical or biological markers of impending symptomatic disease [42, 43]. However, MCI is so heterogeneous in its presentation, underlying pathology and evolution, that it cannot be used as a surrogate marker.

\section{Structural imaging using MRI}

While helpful in supporting the diagnosis of $\mathrm{AD}$, this approach is confounded by the presence of atrophy due to other pathological processes and the generalised atrophy that occurs as part of normal ageing [44, 45]. One way to confront this problem is to use measurements of multiple anatomically specific brain volumes other then the hippocampus and entorhinal cortex that are affected by AD. Examples include: the amygdalae, superior temporal sulcus, cingulate gyrus, temporal-parietal regions and the frontal lobe. This provides the potential to increase the diagnostic power of MRI-derived atrophy measurements by additional anatomically defined areas [46].

Although very high resolution computed tomography can be used to image neuritic plaques in experimental animals [47], it is currently not possible to accomplish this in a clinical setting. However, by modifying existing clinical MRI acquisition protocols to incorporate measures of $\mathrm{T}_{1}$ relaxation time, it is possible to quantify the accumulation of abnormal protein aggregates in anatomically defined brain regions. This opens the possibility of developing an MRI-based molecular imaging BMs for $\mathrm{AD}$ related pathology that could be used in healthcare facilities [48].

Proton MR Spectroscopy ( ${ }^{1} \mathrm{H}$ MRS) is a diagnostic imaging technique that is sensitive to changes at the cellular level. With ${ }^{1} \mathrm{H}$ MRS, several of the major proton-containing metabolites in the brain can be measured during a common data acquisition period. The metabolite $\mathrm{N}$-acetyl aspartate (NAA) is a marker for neuronal integrity. NAA decreases in a variety of neurological disorders including clinically diagnosed $\mathrm{AD}[49,50]$. The decrease of NAA or the $\mathrm{NAA} /$ creatine $(\mathrm{Cr})$ ratio was observed in the posterior cingulate of $\mathrm{AD}$ patients $[51,52]$.

In patients with mild to moderate $\mathrm{AD}, \mathrm{NAA} / \mathrm{Cr}$ levels are lower than normal in the medial occipital lobe, including the visual cortex. This regional pattern is in agreement with the distribution of the neurofibrillary pathology, and associated neuron loss in people with mild to moderate $\mathrm{AD}$, indicating that $\mathrm{NAA} / \mathrm{Cr}$ levels are potential surrogates for early detection of $\mathrm{AD}$ and for disease progression [53].

Another metabolite peak of interest in the ${ }^{1} \mathrm{H}$ MRS of the brain in $\mathrm{AD}$ is the choline (Cho) peak. Some studies identified elevated Cho and $\mathrm{Cho} / \mathrm{Cr}$ ratios, while some reported normal levels in people with $\mathrm{AD}$ compared to normal [54]. The largest amount of choline in the brain is in choline-bound membrane phospholipids that are precursors of choline and acetylcholine synthesis. It has been postulated that the elevation of Cho peak is the consequence of membrane phosphatidylcholine catabolism to provide free choline for the chronically deficient acetylcholine production in AD [55]. This appears to be useful in detection of presumed pathologic changes in people with clinical diagnosis of AD. Lower, NAA levels, NAA/Cho has also been observed at the preclinical or MCI stage [56].

\section{PET and SPECT}

$\mathrm{AD}$ is associated with metabolic impairments that often have a 'typical' pattern, initially affecting the parietal and temporal lobes as well as posterior cingulate cortex before progressing to the frontal lobes and other brain structures. These metabolic activity can be visualized using $\left[{ }^{18} \mathrm{~F}\right]-2$ fluoro-deoxy-D-glucose PET and the pattern of involvement is often useful in distinguishing AD from other LLD [57, 58] (Fig. 1).

The impaired metabolic activity may, in part, be due to a loss of neurons in these areas and in part may be a sign of reduced activation, for example reflecting synaptic loss [59]. In any case, it is not the mere loss but rather its regional distribution which characterise AD. However, while the distribution of metabolic changes or even atrophic changes may have typical distributions, these are rarely highly specific. The observed changes, e.g. fronto-temporal atrophy or hypo-metabolism associated with primary progressive aphasia-PPA, for example may reflect a large number of underlying pathological processes (e.g. AD, Frontotemporal dementia-FTD).

Evidence exists that the pattern of temporal and parietal hypometabolism and hyporepfusion are reasonably sensitive and specific to $\mathrm{AD}$. Comparing $\mathrm{AD}$ patients to controls showed relatively high sensitivity and specificity (85-90\% respectively) in differentiating $\mathrm{AD}$ patients from controls $[60,61]$. Although differentiation of AD from normal ageing seems clinically irrelevant, the evidence for the ability of PET and SPECT to detect functional abnormalities in very mild dementia has considerable importance in the early diagnosis of dementia [60-62]. A recent European multi center PET study that used quantitative FDG-PET methodology found $93 \%$ sensitivity and $93 \%$ specificity in differentiating $\mathrm{AD}$ patients from controls when patients with Mini Mental State Examination score of 24 or greater were included [63].

More recently radioactive ligands have been developed to visualise aggregation of amyloid-beta $(A \beta)$ peptides. 


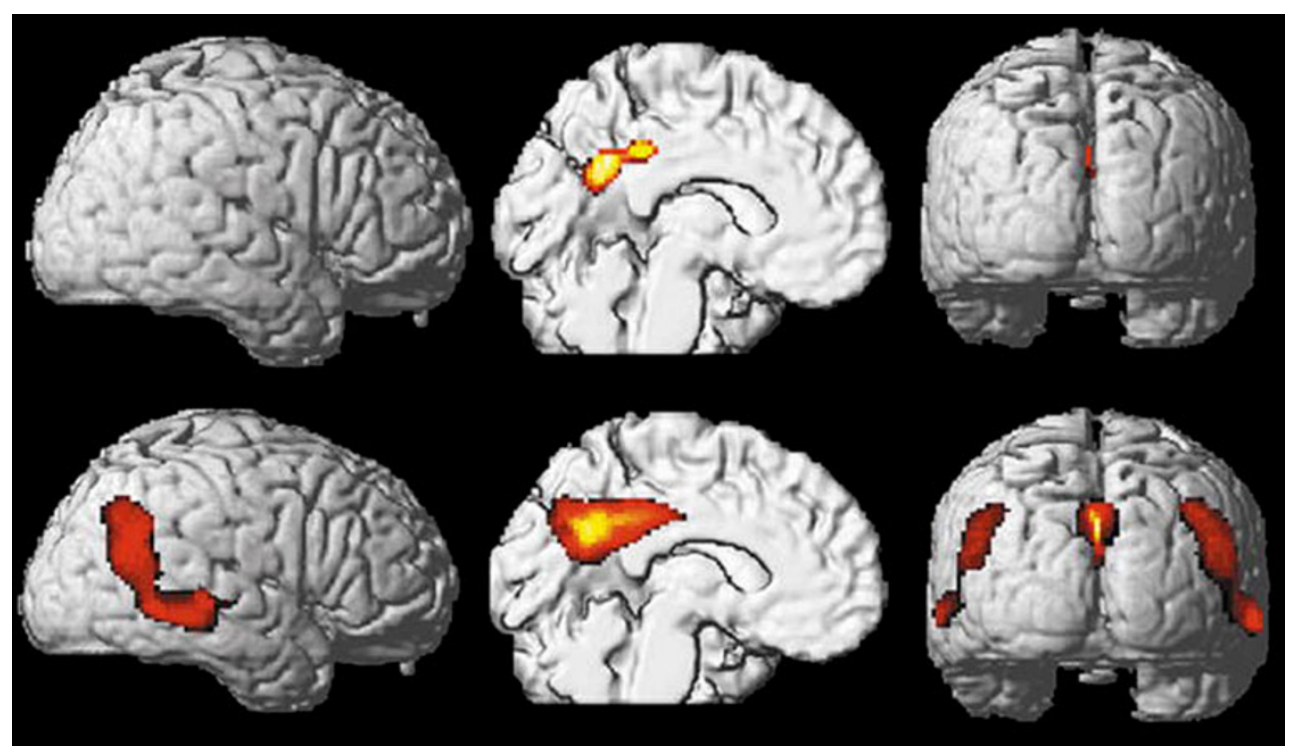

Fig. 1 Brain hypometabolism in $\mathrm{MCI}$ and $\mathrm{AD}$. Brain regions showing reduced glucose metabolic rate (MRglc) in amnestic MCI (top row) and $\mathrm{AD}$ (bottom row) in comparison with matched normal controls. The cortical MRglc reductions in an amnestic MCI group $(n=37)$ were found to be restricted to the posterior cingulate cortex, while they were spatially more extended and involved several regions of the association cortex in an $\mathrm{AD}$ group $(n=87)$. The distribution of MRglc reductions in MCI suggests that these patients share some common feature with $\mathrm{AD}$ patients. Statistical parametric maps are superimposed onto the right lateral (left), medial (middle) and posterior (right) views of a volume-rendered spatially normalised MRI study $(p<0.001$, uncorrected for multiple comparisons) Taken with permission from [64]

imaging diagnosis has not yet been shown to supplement a clinical evaluation in any circumstance. Another approach is therefore to compare clinical and imaging diagnosis in the same sample, asking whether imaging provides diagnostically useful information in addition to the clinical data [59]. In this regard it is worth mentioning the Alzheimer's Disease Neuroimaging Initiative (ADNI) whose primary goal is to determine whether brain imaging, biochemical markers, and clinical and neuropsychological assessment can accurately measure the progression of mild cognitive impairment and early AD. The ADNI cross-sectional and longitudinal study has successfully recruited more than 800 individuals, normal controls, subjects with MCI, and subjects with mild $\mathrm{AD}$, followed for 12 months, that were very similar to those seen in MCI and mild AD clinical trials [71]. The data from this clinical trial, which can be accessed in a public website, are of utmost relevance to researchers and clinicians for helping diagnose $\mathrm{AD}$, develop new treatments and assess their effectiveness in the quest to improve safety and decrease the time and cost of clinical trials. Importantly, the associations of the genetic data with the neuroimaging, neurological and biochemical patterns detected in the ADNI data will allow the survey of genetic factors related to the rate of progression of Alzheimer's disease.

Lastly, the diagnosis needs a cheap and fast BM, and none of the imaging methods mentioned above can do that. 
Biochemical markers—-serum and cerebrospinal fluid

Most of the CSF is produced at the plexus choroideus. The CSF then flows from ventricular space to the subarachnoid space and back to the serum through the paccioni granulations. A further fraction of the CSF flows along the spinal cord. Here CSF proteins can reach the serum along the nerve sheaths by passive diffusion [41]. Thus, potentially promising BMs can be seen in the CSF. Lumbar puncture is a relatively safe and uncomplicated procedure for sampling of the CSF, and only a small ratio of $\mathrm{AD}$ patients complain about post-puncture complications [18].

Neurofibrillary protein aggregates containing phosphorylated tau are one of the major hallmarks of AD. In normal cells, tau stabilises axonal microtubules, which are the tracks for intracellular traffic. In AD, tau becomes abnormally phosphorylated, aggregates into paired helical filaments and loses its ability to maintain the microtubule tracks. In the past several years numerous blood and CSF tests have been proposed for early detection of $\mathrm{AD}$ and related disorders. These tests are based on disease pathology. Most common measurements of the CSF regarding $\mathrm{AD}$ focus on the tau protein and $\mathrm{A} \beta$-peptides. $A \beta$-peptides result from enzymatic breakdown of APP by $\alpha-, \beta$ - and $\gamma$-secretases and make up the main part of amyloid deposits in AD [72].

Quantification of tau and $A \beta$ in the CSF represents the most intensively studied BM of AD [73]. Approximately $80 \%$ of patients who meet clinical criteria for $\mathrm{AD}$ have elevated levels of CSF tau [74]. Autopsy studies confirmed this association and validated the relationship between high levels of CSF tau and a pathologic diagnosis of AD [75].

The study by Arai and coworkers [76] addressed the question whether CSF BMs could detect incipient $\mathrm{AD}$ among MCI patients. Results presented by these authors seem to indicate that high CSF total tau (t-tau) could discriminate between memory impaired individuals that later progressed to $\mathrm{AD}$ and those that did not convert. According to the data presented in the manuscript the test demonstrated $90 \%$ sensitivity and $100 \%$ specificity. The results have been recently confirmed [77]. However, since the values of these BMs do not change significantly throughout the course of the disease, they cannot be of utility as biochemical predictors in AD-modifying treatments with anti-acetyl cholinesterase agents, inhibitors of $\beta$ and $\gamma$ secretases or vaccination regimens.

The development of specific phosphate-dependent tau antibodies raised the possibility that $\mathrm{AD}$ could be uniquely identified by quantifying tau phosphorylated in one of several locations: threonine-181 (p-tau181), serine-199 (p-tau199) or threonine-231 (p-tau231). Boban and coworkers [78] tried to determine the diagnostic accuracy of the level of CSF t-tau, p-tau181 and p-tau199 BMs in a group of AD and VaD patients. The results showed that CSF t-tau and p-tau199 were useful markers for differentiating between $\mathrm{AD}$ and $\mathrm{VaD}$. However, while elevated levels of $\mathrm{t}$-tau in the CSF supported the diagnosis of $\mathrm{AD}$, failure to find elevated CSF t-tau reduced, but did not eliminate, the possibility of $\mathrm{AD}$ pathology.

Another line of recent evidence suggests that CSF p-tau231 declines during the natural course of AD. Seventeen pharmacologically untreated patients with $\mathrm{AD}$ were followed up to six years with repeated serial CSF measurements. CSF p-tau 231 concentration, but not $\mathrm{t}$-tau, decreased over time in $\mathrm{AD}$, independent of age. Rate of change was inversely correlated to the Mini Mental State Examination (MMSE) score at baseline and provided objective measure for the cognitive decline [79]. These results suggest that CSF p-tau 231 may have the potential to track $\mathrm{AD}$ progression and may be a valuable tool to map effects of disease modifying drugs on AD specific neurodegeneration.

Neuritic plaques associated with $\mathrm{AD}$ contain $\beta$-pleated sheets of amyloid. The 42-amino-acid fragment of $A \beta_{(1-42)}$ is especially prone to fibrillisation and disproportionally accumulates in extracellular lesions in $\mathrm{AD}$ brains and was found to be reduced in the CSF of AD patients [75, 80]. However similar reductions occur in a variety of other conditions and as a diagnostic BM CSF $\mathrm{A} \beta_{(1-42)}$ provides only a small addition of information beyond what is obtained from the CSF t-tau level [76].

The reduction in CSF $A \beta_{(1-42)}$ in $A D$ has been hypothesised to reflect the deposition of the peptide in senile plaques, with lower levels diffusing to the CSF. Thus, according to Hulstaert and coworkers [81] decrease in $A \beta_{(1-42)}$ in $A D$ is probably the most consistent $B M$ finding. $A \beta_{(1-42)}$ alone showed a sensitivity of $78 \%$ and specificity of $81 \%$ in distinguishing $\mathrm{AD}$ patients from normal elderly controls. Studies correlating CSF A $\beta_{(1-42)}$ protein concentrations with cognitive performance in $\mathrm{AD}$ were partly contradictory. Cross-sectionally, the concentration of $A \beta_{(1-42)}$ protein and cognitive measures were either inversely correlated [82] or no significant correlation was found [81]. In a longitudinal study, a decrease of CSF $\mathrm{A} \beta_{(1-42)}$ protein has been found within a 3 year follow-up [83]. A highly significant correlation between low CSF concentrations at baseline and 1 year serial follow-up was found. There was no correlation between CSF levels and duration or severity [18]. The potential value of $A \beta_{(1-42)}$ protein during the course of $\mathrm{AD}$ progression should be further evaluated.

Others [84] criticised that such differences might be the result of differences in patients and control material. Furthermore, low CSF levels of $A \beta_{(1-42)}$ were also found in Creutzfeldt-Jakob disease [85, 86], DLB [87] and amyotrophic lateral sclerosis (ALS) [86], thus making the 
ability of $A \beta_{(1-42)}$ to distinguish between $\mathrm{AD}$ and other neurological disorders relatively limited.

In addition to CSF BMs, studies by others have focused on plasma BMs. For example, the study by Ringman and coworkers [88] provided preliminary support for a model in which BMs can predict disease progression in presymptomatic familial AD persons. Participants underwent evaluations for presenilin 1 or APP mutations and cognitive evaluations with Clinical Dementia Rating Scale (CDR). The data obtained in this study indicated that plasma $A \beta_{(1-42)}$ is elevated in familial $A D$ mutations carriers and that this level may decrease with the cognitive decline of disease progression prior to the development of dementia. These results are in conflict with those found by Hulstaert and coworkers [81].

In one study [89] plasma $A \beta$ levels were found to be elevated in EOAD (Early Onset AD) as well as in sporadic LOAD and in first degree relatives of patients with LOAD. This finding provides evidence for novel and yet unknown factors that lead to elevation of plasma $A \beta$ in asymptomatic young individuals from LOAD families.

Promising novel $\mathrm{BMs}$ for $\mathrm{AD}$ have been recently documented by Ray and coworkers [90] focusing on the expression of 18 plasma signalling and inflammatory proteins, out of 120 , that predicted the progression of patients who had MCI to AD 2-6 years later. Using a proteome-based approach [91], the authors characterised a number of proteins previously implicated in the disease pathology of $\mathrm{AD}$ in plasma versus control patients, including complement factor $\mathrm{H}(\mathrm{CFH})$ precursor and alpha-2-macroglobulin (alpha-2 M), whose expression correlated with disease severity. Other BMs (e.g. isoprostanes) have also been studied in the plasma [92, 93]. However, further studies aimed at investigating plasma BMs would be necessary to improve sensitivity, specificity and replication in independent institutions, to evaluate their diagnostic value.

\section{Biomarkers for Parkinson's disease}

Aetiology and pathogenetic mechanisms of PD

PD may be the second most common neurodegenerative disease after $\mathrm{AD}$, featuring an incidence that increases with age and a higher prevalence throughout the male population. PD is a multifactorial disease and several factors related to genes, age, sex, and environment may increase the risk of the disease. A small minority of all PD cases is of genetic origin [94, 95]. Mutations in alpha $(\alpha)$-synuclein, parkin, ubiquitin c-terminal hydrolase (UCH)-L1 and DJ-1 have been linked to juvenile forms of PD, with an early onset before the age of 40 years. For details see Table 1 and section Genetic markers of PD below. Several pesticides such as paraquat, maneb, dieldrin, eptaclor and the more recent rotenone have been associated with an increased incidence of PD among farmers. Moreover, a positive association between exposure to heavy metals including manganese, mercury, lead and PD has been reported [96].

The cardinal symptoms of PD include bradykinesia, hypokinesia and akinesia, muscular rigidity and resting tremor, often progressing to postural instability, gait problems and freezing. More recently, considerable attention is given to a myriad of non-motor symptoms related to autonomic disturbances, such as hypotension, constipation, bladder and thermoregulatory dysfunctions and sleep disturbances. PD may also be associated with olfactory dysfunction, depression and anxiety together with the onset of cognitive deficits and dementia as the disease progresses [97].

Table 1 Identified genes linked to familial PD

\begin{tabular}{lllll}
\hline Locus & Gene/protein & Chromosome location & Inheritance & Suggested function \\
\hline PARK 1/4 & SNCA/ $\alpha$-synuclein & $4 \mathrm{q} 21-\mathrm{q} 23$ & AD & Synaptic function/vesicle trafficking \\
PARK 2 & parkin & $6 \mathrm{q} 25-\mathrm{q} 27$ & AR & E3 ubiquitin ligase \\
PARK 5 & UCH-LI & $4 \mathrm{p} 14$ & AD & Ubiquitin C-terminal \\
PARK 6 & PINKI & $1 \mathrm{p} 35-\mathrm{p} 36$ & AR & Mitochondrial serine/threonine kinase \\
PARK 7 & DJI & $1 \mathrm{p} 36$ & AR & Chaperone, oxidative stress response \\
PARK 8 & LRRK2/dardarin & $12 \mathrm{p} 11.2-\mathrm{q} 13.1$ & AD & Protein kinase \\
PARK 9 & ATP13 A2 & $1 \mathrm{p} 36$ & AR & Lysosomal type 5 ATPase \\
PARK 13 & OMI/HTRA2 & $2 \mathrm{p} 12$ & AD & Serine protease \\
PARK14 & PLA2G6 & $22 \mathrm{q} 13.1$ & AR & A2 phospholipase \\
PARK 15 & FBXO7 & $22 \mathrm{q} 12-\mathrm{q} 13$ & AR & SCF E3 ubiquitin ligase component \\
Not assigned & NR4A2/NURR1 & 2q22-q23 & Unknown & Probable nuclear receptor \\
Not assigned & Synphilin-1 & $5 \mathrm{q} 23.1-\mathrm{q} 23.3$ & Unknown & Synaptic function, protein degradation \\
\hline
\end{tabular}

$A D$ autosomal dominant; $A R$ autosomal recessive 
The pathological hallmark of PD is represented by degeneration of the substantia nigra (SN) dopaminergic neurons associated to the presence of intra-cytoplasmatic inclusions of ubiquitin and $\alpha$-synuclein denominated Lewy bodies (Fig. 2). It is interesting to note that the motor symptoms characterising the disease are manifested once degeneration of the dopaminergic nigro-striatal pathway has reached at least $50-60 \%$ [98]. This observation is in accordance with the notion that there is a prolonged period that precedes motor symptoms manifestation and is characterised by an extensive non-dopaminergic pathology, which involves several other neurotransmitters, including acetylcholine, noradrenaline, serotonin, glutamate and adenosine [99]. These abnormalities are responsible for the non-motor pathology of PD that may precede the onset of motor symptoms, sometimes by years and are often considered more debilitating to the afflicted patients. Common manifestations are freezing, falling, cognitive decline and the aforementioned dysfunctions such as anosmia (loss of sense of smell), anxiety, and depression [100]. This receives support from the post-mortem study of Braak et al. [101] who mapped the sequence of disease progression from brainstem to the basal ganglia and cortical regions, according to advances observed in the accumulation of $\alpha$-synuclein-immunopositive Lewy bodies in different brain structures. Further data suggest that PD pathology may even originate in peripheral nerve tissues as the mesenteric plexus in the gut and perhaps cardiac neurons [102, 103].

It is apparent that the damage to $\mathrm{SN}$ pars compacta occurs later in the course of the disease, while other brain areas and peripheral tissues are initially affected at the presymptomatic phase of the disease. Clearly, the identification of suitable valid biological markers that can be monitored from preclinical stages in asymptomatic 'at-risk' subjects, will allow the capturing of high-risk individuals in the valuable therapeutic window of about five years before the appearance of clinical signs.

Currently, the diagnosis and outcome measures of PD rest on the physician's physical examination scored with the Unified Parkinson's Disease Rating Scale (UPDRS) [104], the modified Hoehn and Yahr (H\&Y) staging scale [105] and neuroimaging assessment (Fig. 3 and section Neuroimaging markers below), which, however do not always correlate. Although a diagnosis of PD can be relatively accurate in patients with a typical presentation of cardinal signs and response to levodopa treatment, the differential diagnosis versus different forms of parkinsonism may have greater overlap and thus misdiagnosis can occur in up to $25 \%$ of patients [106]. Thus, there is hope that a combination of different BMs (imaging, biochemical and genetic) will help diagnose symptomatic disease stages and complement the clinical outcome in clinical studies of disease modifying therapies.
Fig. 2 Substantia nigra degeneration in PD and dementia with Lewy bodies. The core pathology of PD affects the DA-producing neurons of the substantia nigra $(\mathrm{SN})$. DA is produced by $\mathrm{SN}$ neurons and transported along the axons of these neurons to the striatum. The triad of rigidity, bradykinesia and tremor at rest, correlates with degeneration of the dopaminergic nigrostriatal pathway and DA depletion in the striatum. In advanced PD, loss of these neurons results in depigmentation of the SN (A) and in loss of the DA synthesising enzyme tyrosine-hydroxylase (TH) (BC). PD is a synucleinopathy. Fibrils made of insoluble polymers of $\alpha$-synuclein are deposited in the neuronal body, forming round lamellated eosinophilic cytoplasmic inclusions, the Lewy bodies (D). $\alpha$ synuclein is also deposited in neuronal processes

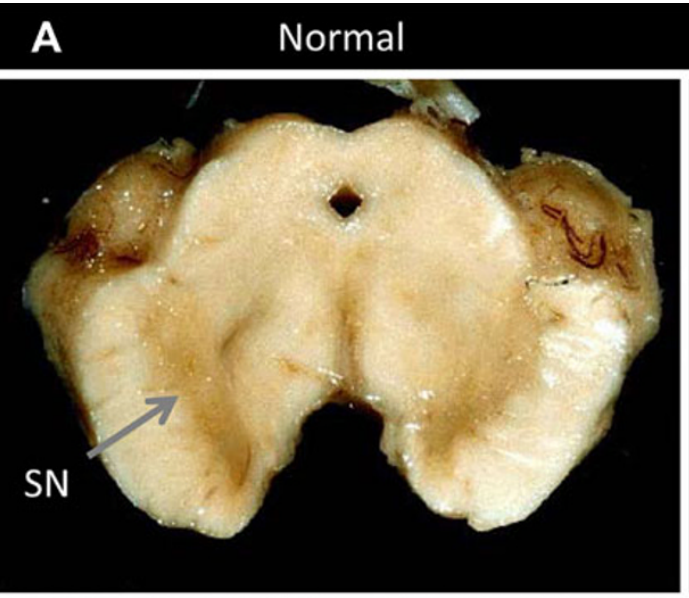

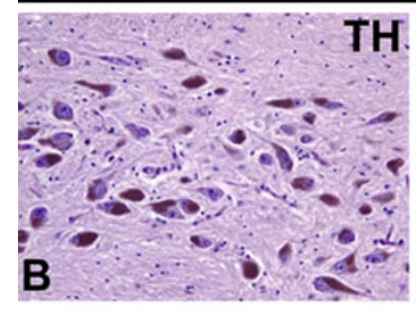

Normal SN parscompacta

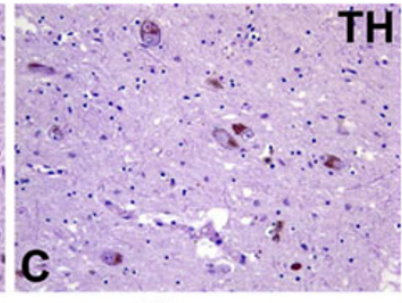

SN parscompacta in PD

$\mathrm{TH}$
Parkinson's disease
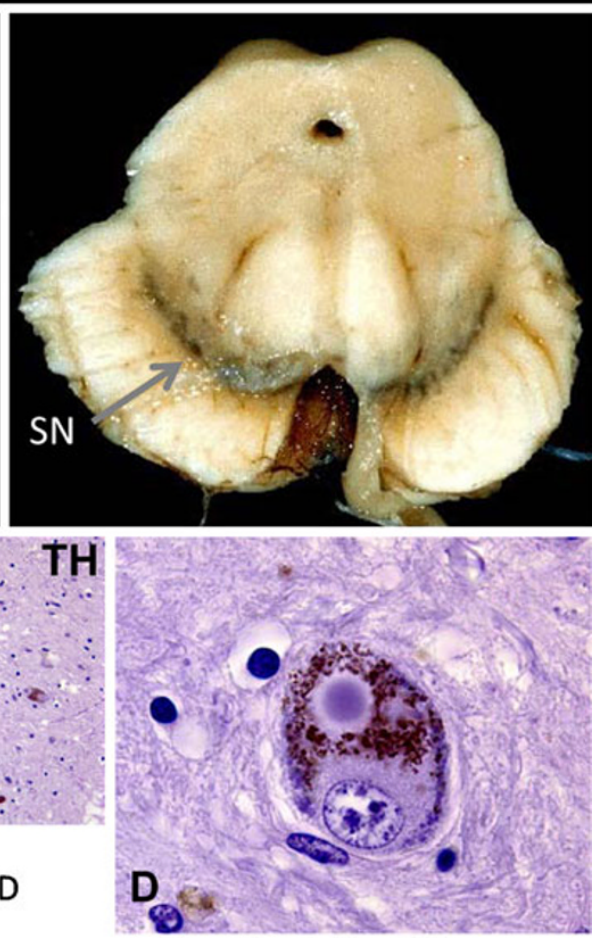

Lewy body in a SN neuron 
Fig. 3 Imaging findings of the presynaptic dopaminergic system in PD. (A) Colour-coded diffusion-weighted MRI and (B) striatal 123I-2 $\beta$-carbomethoxy$3 \beta$-(4-iodophenyl)-N-(3iodophenyl)tropane uptake for a healthy individual, a patient with $\mathrm{PD}$, and a patient with the atypical parkinsonian syndrome multiple system atrophy (MSA). The apparent diffusion coefficient (A) is normal in the striatum in PD but it is raised in MSA (arrows) because of the neuronal loss that targets the putamen. DA transporter binding $(\mathbf{B})$ is bilaterally reduced in the striata in both PD and MSA. In $\mathrm{PD}$, the caudate is relatively spared compared with the putamen. Taken with permission from [142]
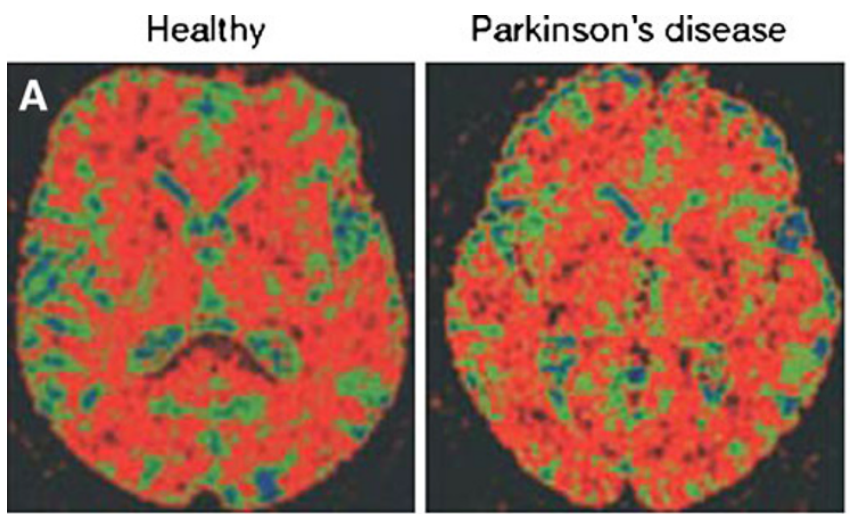

Multiple system atrophy
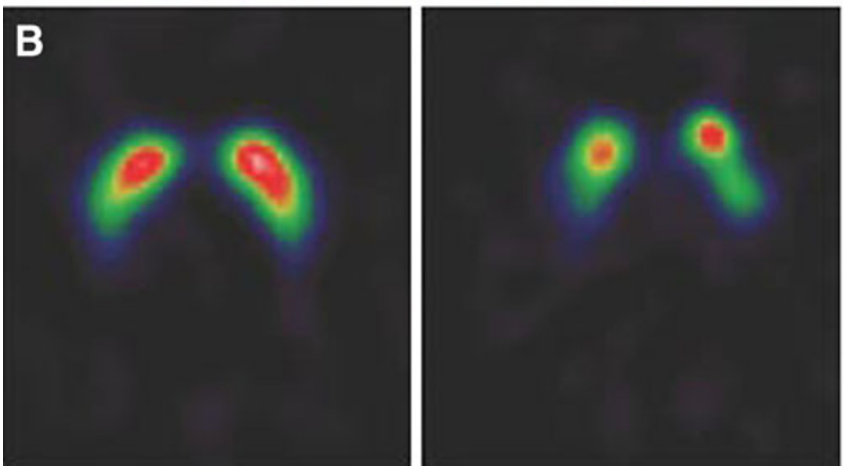

Obviously, misdiagnosis can lead to inappropriate therapy, for example prescribing cholinesterase inhibitors to patients with an underlying pathology, such as PSP.

\section{Predictive behavioral clinical tests}

Symptoms including sleep disturbances, mood disorders and olfactory and autonomic dysfunctions (e.g. constipation) have been identified during both the pre-motor and motor symptomatic phase of the disease. Accordingly, the presence of these dysfunctions may contribute towards the clinical diagnosis of PD.

Presence of sleep disturbance with reduced rapid eye movements (REM) sleep and frequent and prolonged waking throughout the night is commonly associated with PD. REM sleep behavior disorder in patients affected by PD is closely related to incipient cognitive impairment, whereas quantitative motor testing, olfactory deficits and autonomic symptoms are not [107, 108]. Reduced REM sleep latency (time from sleep onset to the first REM episode) was found in a high proportion in PD patients, however its incidence was significantly higher in depressed patients suggesting that differences in latency may be related to depression rather than PD.

Depressed mood is often associated to PD and several recent studies have confirmed that depression in the PD patient is a major determinant of quality of life [109]. Depression in PD might be associated with a specific loss of dopamine (DA) and noradrenaline innervation in the limbic system [110]. Interestingly, several studies have suggested that depression in the PD patient is associated with a more rapid deterioration in cognitive functions [109].

Olfactory deficits reported in PD are thought to represent a sensitive marker of the disease which could allow an early diagnosis and help in differentiating idiopathic PD from other diseases with features of parkinsonism. The diagnostic accuracy of olfactory testing in differentiating PD from other disorders is however insufficient to justify routine clinical use [111]. Olfactory dysfunctions are often measured by means of the University of Pennsylvania Smell Identification Test (UPSIT). Major deficit in olfactory judgement is present in PD, suggesting that this deficit is associated with disruption of olfactory areas situated in the temporal lobes and the prefrontal cortex [112], although olfactory bulb changes may have an important role.

Diagnostic test battery Montgomery et al. [113] developed a behavioral diagnostic test battery in order to provide with early diagnosis of PD. The test battery incorporates tests for motor, olfaction and mood evaluation. As mentioned above, symptoms of olfactory dysfunction and depression often antedate the diagnosis of PD; addition of the wrist flexion and extension movement test provided a test battery featuring high specificity $(88 \%)$ and sensitivity rate $(69 \%)$, not previously achieved by any test alone. The latter battery test has been successfully applied in the prospective identification of as yet undiagnosed patients 
with signs suggestive of PD who have been subsequently diagnosed as affected by the disease [114].

MCI Based on findings reported in several studies on PD featuring dementia and DLB, Troster [115] performed a critical evaluation to determine whether MCI may constitute an early symptom of PD with dementia and whether it may prove possible to differentiate between PD with dementia or DLB. The conclusions drawn in the review underline how, at present, the data available do not allow an adequate evaluation of the utility of MCI, which for PD mainly concerns executive functions, as a predictive test for PD with dementia. However, the author acknowledged that to date scarce attention has been devoted to cognitive impairment and the role of depression on the latter. Moreover, DLB and PD with dementia, although very similar from several standpoints, should be viewed as two separate entities [115].

Autonomic dysfunctions including orthostatic dizziness, constipation, bladder dysfunction, pain, erectile dysfunction and hyperhydrosis are associated to PD and appear as early symptoms prior to the onset of motor disorders [116, 117]. A new diagnostic strategy utilising skin biopsy developed by Sommer et al. [118] and applied to PD patients by Rossi et al. [119] suggests that small peripheral fibers may be reduced in PD patients. Analysis of small caliber sensory nerves might therefore represent a suitable approach for use in the characterisation of early autonomic dysfunctions in PD.

\section{Genetic markers of PD}

Genes causing PD Parkinsonism has been long viewed a 'sporadic' disorder although a familial component was noted over a century ago by the studies of Leroux in 1880 [120] and Gowers in 1900 [121], who first suggested that heritable factors might increase disease susceptibility. It was not until the last decade that the contribution of genetics to familial forms of PD became much greater acknowledged. At present, fifteen genomic chromosomal regions have been described and five pathogenic mutations in genes unequivocally linked to familial PD have been identified: the autosomal dominant LRRK2 and SNCA and autosomal recessive Parkin, PINK1 and DJ1 [94, 122] (Table 1).

Dominantly-inherited PD Genetic studies of PD began with the discovery of mutations in the gene SNCA that encodes $\alpha$-synuclein. Three point mutations have been identified that segregate with familial PD, Ala53Thr [123], Ala30Pro [124] and Glu46Lys [125]. Patients carrying SNCA mutations clinically respond to levodopa, although disease onset is earlier than in patients with idiopathic $\mathrm{PD}$, and progression appears to be more rapid. Neuropathologically, SNCA carriers display similar features as seen in idiopathic disease including cell degeneration, Lewy bodies, and neuritic staining. Notably, not only missense mutations, but also multiplications (duplication and triplication) of SNCA cause PD with a clear effect of dosage on phenotypic severity in terms of age of onset, disease duration and severity [126-128].

A mutation in the ubiquitin carboxy-terminal hydrolase L1 gene (UCH-L1, PARK5) was detected in a single sibling pair in a German family resembling typical PD with disease onset around 50 years of age [129]. UCH-L1 has ubiquitin hydrolase and ligase activities thus playing a role in the ubiquitin proteosomal system (UPS) which degrades damaged proteins system. This and its reported localisation in the Lewy bodies [130] support the suggested link between the UPS, endosomal-lysosomal pathways and Lewy body in late-onset PD, posing UCH-L1 a convincing candidate gene for PD.

Mutations in the leucine-rich repeat kinase 2 (LRRK2) gene (PARK8) are the most common genetic determinant of PD identified to date and have also been found in late-onset PD patients without a known family history of PD, suggesting a significant genetic component [94, 95]. LRRK2 mutant PD is estimated to account for approximately $7 \%$ of familial PD cases and up to $3 \%$ of apparently sporadic disease, although significant ethnic differences exist. Accumulating evidence indicate that the G2019S mutation in LRRK2 and homozygous or compound heterozygous mutations in the lysosomal enzyme $\beta$ glucocerebrosidase (GBA), the enzyme that is deficient in Gaucher's disease, are among the most prevalent genetic risk factors for PD. These mutations are most common among the Ashkenazi-Jewish population [131, 132]. $L R R K 2$ encodes a putative protein kinase and is predicted to be involved in multiple functions in view of the diverse protein domains which include a GTPase and kinase domain, as well leucine-rich and WD40 repeats protein interaction domains. Besides pathogenic mutations, variants in LRRK2 have been reported that increase the risk for sporadic PD [122] (Table 1).

Recessively-inherited $P D$ Four recessive forms of $\mathrm{PD} /$ parkinsonism have been identified in Parkin (PRKN, PARK2) [133], PTEN-induced kinase 1 (PINK1, PARK6) [134], DJ1 (PARK7) [135] and ATPase type 13A2 (ATP13A2, PARK13) [136]. These mutations are relatively rare, with probable loss-of-function mechanisms, resulting in early age of onset ( $<$ age 45 years).

Mutations in the Parkin gene were originally identified in Japanese families with autosomal recessive, juvenile parkinsonism [133]. Parkin has been accredited to function 
as an E3 ubiquitin-ligase, targeting proteins for degradation by the proteasome [137]. While several parkin-interacting proteins have been identified as putative targets for Parkin ubiquitination, their pathological role in the death of the SN pars compacta neurons is still elusive.

PINK1 contains a serine/threonine protein kinase domain [134], and an N-terminal mitochondrial targeting sequence that localises the protein to mitochondria. Recently, PINK1 has been demonstrated to regulate phosphorylation of another PD-linked gene, Omi/HtrA2 (PARK13) modulating the response to cellular OS [138] and to act in a common pathway with Parkin in maintaining mitochondrial integrity and function in both muscles and dopaminergic neurons [139].

$D J 1$ encodes a mitochondrial protein putatively operating as an OS sensor within cell. DJ-1 mutations are rare, accounting for less than $1 \%$ of early-onset PD. The phenotype of an early-onset Parkinsonism is comparable with Parkin- and PINK1-linked forms of the disease [94].

Mutations in the $A T P 13 A 2$, a protein with lysosomal localisation have been recently linked to autosomal recessive parkinsonism in families with Kufor-Rakeb syndrome [136]. Patients have juvenile onset atypical parkinsonism, accompanied by pyramidal cell degeneration and dementia.

Collectively, the discovery of genes linked to heritable forms of PD provides potential main targets for therapeutic intervention. PINK1, DJ1, parkin and OMI/HTRA2 are all associated with the mitochondria and have been implicated in protection against oxidative stress, alluding to a common pathway in their cell mechanism of action. The malfunction of these proteins may compromise mitochondrial integrity with subsequent increased levels of free radicals, reduced cellular energy and eventual impaired UPS function and protein aggregation, further supporting a link between mitochondrial impairment and the pathogenesis of PD. Thus, modulation of the function of Parkin, PINK1 or DJ-1 or interfering with the function of LRRK2 or with the expression and accumulation of $\alpha$-synuclein may hold promise for neuroprotection in PD.

In addition to the familial PD-linked genes, several genomic loci have been designated in linkage studies as either being linked or influencing the age of onset of PD, such as microtubule-associated protein tau (MAPT) and UCHL (for review of candidate genes studied today see [94, 95, 122]). Current large-scale genome-wide association studies will extend genome coverage in order to reveal common PD susceptibility genes.

\section{Neuroimaging markers}

Imaging PD involves either detecting alterations in brain structure or examining functional changes in brain meta- bolic systems. In PD, degeneration of the dopaminergic system is accompanied by cholinergic, noradrenergic, and serotonergic dysfunction. Function of these dopaminergic and non-dopaminergic systems can be imaged with PET and SPECT and correlated with motor and non-motor disturbances. Dopa decarboxylase activity at DA terminals and DA turnover can both be measured with ${ }^{18} \mathrm{~F}$-dopa PET. Presynaptic dopamine transporters (DATs) can be followed with PET and SPECT tracers such as ${ }^{123}$ I- $2 \beta$-carbomethoxy$3 \beta$-(4-iodophenyl)-N-(3-iodophenyl)tropane while vesicle monoamine transporter (VMAT) density in DA terminals can be examined with ${ }^{11} \mathrm{C}$-dihydrotetra-benazine $\left({ }^{11} \mathrm{C}\right.$ DTBZ) PET (Fig. 3).

Measurements of DA terminal function can sensitively detect DA deficiency in both symptomatic patients and individuals at risk for parkinsonian syndromes [140], but have poor specificity for discriminating between typical (idiopathic) and atypical PD (e.g. head trauma, druginduced parkinsonism, PSP, MSA). On the other hand, measurements of glucose metabolism with ${ }^{18} \mathrm{~F}$-fluorodeoxyglucose PET can be very helpful as normal or raised levels were found in the lentiform nucleus of PD but levels were reduced in MSA and PSP [141].

MRI and transcranial sonography (TCS) can reveal brain structural changes such as volumetric reduction and hyper echogenicity of certain midbrain and striatal areas in patients with PD[142]. TCS and MRI might be particularly valuable for revealing a susceptibility to $\mathrm{PD}$, although they correlate less well with either clinical status or loss of DA terminal function in the striatum (see next section). By contrast, PET and SPECT measurements of DA terminal function do correlate significantly with clinical disability [140].

Although the available BMs for PD are promising, none of them can predict the disease with a high percent of certainty, thus it seems that the best diagnosis will require a combination of markers and clinical assessment.

Imaging of iron: a potential biomarker in neurodegenerative diseases and ageing There is increasing evidence that iron is involved in the processes that underlie many neurodegenerative diseases $[143,144]$. Iron-related neurodegenerative disorders can be separated into those that result from iron accumulation in specific brain regions, and those that result from defects in iron metabolism and/or homeostasis. Normally, the total iron level in the brain of newborns is quite low, but increases rapidly until stable levels are reached at an age of 20-30 years [145]. Non-haem iron (mostly ferritin) progressively accumulates particularly in regions that are affected by $\mathrm{AD}$ and $\mathrm{PD}$ such as the putamen, motor cortex, prefrontal cortex and thalamus during the first 30-35 years of life, and variable changes are observed in older individuals [144, 146]. Furthermore, 
brain areas responsible for motor functions (extrapyramidal regions) appear to have more iron than non-motor-related regions, which might explain why movement disorders are commonly associated with iron imbalance [143, 145].

A quantitative evaluation study has revealed deposits of iron (III) in microglia, oligodendrocytes, astrocytes located close to neurons, non-pigmented neurons and in the rim of Lewy bodies in the SN pars compacta of patients with PD. A similar picture of iron accumulation in glia and neurons was found in the putamen and pallidum of the same patients [147, 148]. Significant amounts of iron are sequestered in neuromelanin granules of dopaminergic and noradrenergic neurons of the human SN and locus coeruleus [149]. In light of the aforementioned findings there is a growing conviction that iron distribution and levels in brain areas of PD patients might indicate a degree of vulnerability to PD.

Recent structural neuroimaging studies applying noninvasive TCS have shown that more than 90\% of PDpatients show hyperechogenicity of the SN compared to healthy individuals [150] and this could be related in part to an increased iron content of the SN [151]. Even more, in young healthy adults which presented increased echogenicity of the $\mathrm{SN}$, a functional impairment of the nigrostriatal system was evident. Thus, TCS technique may identify a susceptibility marker that can be detected prior to the clinical manifestations of PD.

\section{Biochemical markers}

As mentioned above, no laboratory tests for use in the detection of blood or CSF BMs correlating with sporadic forms of PD are currently available. We present here a series of BM tests, the clinical application of which needs to be investigated, together with other tests already applied in clinical settings, for the above purpose.

A pioneer study performed by Harrington and Merril [152] described differences in CSF protein pattern in PD patients compared to healthy controls. Subsequently, additional studies performed on lymphocytes or platelets of PD patients have detected subtle abnormalities in $D A$ signaling [153, 154] or mitochondrial functions [155-158], which constitute hallmarks of PD. None of the latter abnormalities, however, has reached the phase of clinical screening.

Activation of glial cells has been constantly found in the brain of patients affected by PD, it is therefore of great interest the variations in prostaglandin D synthase isoforms (PTGDs) described by Harrington [159], since it reflects pathophysiology of meninges and glia, which are a source of prostaglandin D. The reported reduction of the PTGD variant 3 and 4 in CSF of PD patients, measured through 2D gel electrophoresis and mass spectrometry, correlated to changes described in glial cells by several studies, suggesting the suitability of these isoforms as candidate diagnostics markers having a predictive value.

New potential BMs involving the purine system have been recently reported. The first one, linked to a reduced risk of $\mathrm{PD}$, is the concentration of serum urate, the main end product of purine metabolism [160, 161]. Uric acid appears to be first molecular factor directly linked to the progression of typical PD as revealed by a prospective trial showing an inverse correlation of urate levels with clinical and radiographic progression of PD [162]. Urate exerts an antioxidant efficacy comparable to that displayed by ascorbate; accordingly, the high levels registered may act as a defense against oxidative damage, one main acknowledged feature of PD. Urate is implicated in cerebrovascular diseases, therefore, rather than being utilised as a neuroprotective agent, its blood levels may serve as an indirect marker of neuroprotection in neurodegenerative diseases such as PD. More recently, a metabolomic profiling of blood plasma from PD patients has shown a differential expression of analytes related to oxidative damage, lower uric acid and increased glutathione [163]. Thus, urate may be of use in neuroprotective trials as an index of disease progression [162, 164]. Presence of adenosine A2A receptor alterations in putamen of PD patients that mirrors up-regulation in human peripheral blood elements has been also reported. A statistically significant linear correlation among the $A 2 A$ receptor density or tumour necrosis factor-alpha (TNFalpha) levels and UPDRS motor score was described by Varani and coworkers [165]. The correlation found between $\mathrm{A} 2 \mathrm{~A}$ receptor density and UPDRS motor score highlights the central role of $\mathrm{A} 2 \mathrm{~A}$ receptor antagonists in the pharmacological treatment of PD [166].

Another interesting field of potentially useful BMs for $\mathrm{PD}$ is the differential expression of complement proteins [167]. Several types of unidentified proteins present in blood serum have been studied by means of quantitative 2D gel electrophoresis as BMs for use in the diagnosis of neurodegenerative diseases [167, 168]. By using this technique, 34 different serum proteins have been identified in PD and ALS, 9 of which belonging to the complement system. Indeed, differences have been reported between the components of complement $C 3 c$ and complement factor $\mathrm{H}$ and $\mathrm{B}$ in affected subjects compared to controls. Differential expression of complement proteins may represent potentially useful BMs for ALS and PD [169]. These findings lend further support to evidence suggesting that inflammatory processes involving complement cascade elements may be involved in the onset of PD.

Sophisticated techniques have been applied to identify and quantify rare proteins in blood or CSF as BMs. CSF is 
used in proteomic search strategy as the best biological fluid for detecting disease BMs, reflecting the state of substances present in the brain. Using CSF of PD patients, Zhang et al. [170] reported on the first large scale proteomic experiment, the presence of novel BMs for PD and $\mathrm{AD}$. Of these $\mathrm{BMs}$, however, only $\mathrm{A} \beta_{1-42}$ and tau proteins displayed different levels among controls, $\mathrm{AD}$ and $\mathrm{PD}$ and between $\mathrm{AD}$ and $\mathrm{PD}$, as expected. In contrast, the remainders: $\beta D N F$, IL- 8 , vitamin $\mathrm{D}$ binding protein, $\beta_{2}$ microbulin, haptoglobin, apolipoprotein A-I, A-II and E were significantly different from healthy controls, but did not differ between PD and AD.

As mentioned above gene mutations have been linked to familial cases of PD. Interestingly, DJ-1 and $\alpha$ synuclein, two critical interacting proteins, displayed modifications in CSF, serum or plasma levels in sporadic PD. Lower CSF levels of $\alpha$-synuclein have been observed in neurological diseases with primary synucleinopathy, such as DLB and PD [171], whereas plasma levels were increased [172]. Interestingly, plasma $\alpha$-synuclein was found elevated early in the development of PD and DA agonists treatment did not change its plasma levels [173]. These studies have described interesting methods for use in the quantification of $\alpha$-synuclein in biological fluids in diseases characterised by synucleinopathy. Yet, serum levels of alpha-synuclein are unlikely to be a diagnostic marker since in addition to the brain, it is produced in peripheral organs, blood cells and skin [174, 175]. No differences were reported in serum $D J-1$ levels measured in PD patients and healthy controls in a study performed by Maita et al. [176], whereas other studies performed in CSF or plasma, reported higher DJ-1 levels in PD patients than controls [177, 178]. Moreover, while upregulation of CSF DJ-1 levels in early stages of PD was more marked compared to levels observed in advanced stages and in controls, plasma DJ-1 levels appeared to be higher in advanced disease. Likewise, these studies revealed similar increases in PD and in neurological diseases with primary synucleinopathy [177, 178]. How DJ-1 and $\alpha$-synuclein in biological fluids may be of use as BMs in differentiating PD from other neurological disorders remains to be established.

For the scope of BMs in neurodegenerative diseases, it should be borne in mind how clinical proteomic and consequently predictive BMs are strongly influenced by pre-analytical factors such as time or condition of storage [179]. Accumulation of altered proteins and impaired protein clearance may underlie the onset of PD. Recent advances made in methodological procedures used in the study of PD-associated proteins, involving the systematic combined use of BMs may lead to findings capable of providing a crucial contribution to enhancing diagnosis of PD.
Transcriptomics signatures as potential markers of PD

The next section will describe potential BMs based on gene expression studies performed in post-mortem brain tissue and blood-derived RNA from sporadic PD.

Gene expression profiling of sporadic PD SN The current unsatisfactory situation with early diagnosis and available measures of neuroprotection in PD has prompted researchers to look for new approaches using smart advanced biotechnology. During the course of the past decade, we are witnessing the advent of high throughput gene and protein techniques which are becoming especially relevant in the neuroprotective field for ageing and neurodegenerative diseases, since they can provide important targets to develop disease modifying therapies that may have an impact on the disease. Recent microarray-assisted large-scale transcriptomic studies in human post-mortem SN from sporadic PD have revealed significant dysregulation of genes from biological processes linked to previously established neurodegenerative mechanisms both in sporadic and hereditary PD [180]. These include protein aggregation, mitochondrial dysfunction, oxidative stress, cell cycle, vesicle trafficking, synaptic transmission, DA metabolism and cell adhesion/cytoskeleton maintenance. The selected number of candidate genes identified by the various research groups may offer clues for potential gene intersections or cross-talks along the dopaminergic neurodegenerative cascade.

Interestingly, all the gene expression profiling studies conducted so far in the SN of PD patients [180], reported a dysfunction in vesicle trafficking and synaptic transmission functional group such as syntaxin-1 (STX1), synaptotagmin-1 (SYT1) and synapsin 1 and $2(S Y N 1,2)$. Also, all the studies revealed a consistent elevation in heat-shock proteins/stress genes, such as the endoplasmic reticulum protein homocysteine-inducible, endoplasmic reticulum stress-inducible, ubiquitin-like domain member 1 (HERPUD1) and DnaJ (Hsp40) homologue subfamily $\mathrm{B}$, members 1 and $5(D N A J B 1 / 5)$, heat shock proteins $\mathrm{A} 1 \mathrm{~A}$ and A1B (HSPA1A, HSPA1B) and heat shock $27 \mathrm{kDa}$ protein 1 (HSPB1) [180].

The main outcome of the above studies is to provide a view into potential disease mechanistic pathways underlying pathophysiology of PD. Considering the multifactorial nature of $\mathrm{PD}$, reflected in the phenotypic and clinical manifestation variability and the heterogeneity in dopaminergic marker imaging, the information obtained from the functional genomics studies will be useful to assess whether the BM would be relevant to the disease process and consequently, to drug specific treatment. 
Gene expression profiling of sporadic PD blood Currently, there is no blood test that can diagnose PD, making the detection of individuals at risk or at earliest stages of PD practically impossible. For a BM to be clinically useful, non-invasive detection in accessible peripheral tissue is desirable. Blood lymphocytes may offer valuable surrogate markers for neuropsychiatric disorders, as they share significant gene expression similarities to less accessible CNS tissues [181]. Despite the differences in embryonic lineage and biological function between blood and brain, this correlation could be explained, in part, by the fact that blood cells circulate through many different body tissues and thus, may be exposed to the same toxic atmosphere surrounding, for instance, the dopaminergic neurons in the $\mathrm{SN}$ pars compacta. Under these circumstances, the blood cells can alter their transcriptome in response to pathological perturbations, acting as "sentinels", as proposed recently [182]. Regardless of which disease is investigated, the most precise results will be obtained for a carefully selected subset of genes that show similar expression profiles across blood and CNS tissues [181]. In a transcriptome-wide scan study in whole blood tissue from early-staged PD individuals and age-matched controls, Scherzer et al. [183, 184] have found a panel of genes that may modify PD risk. The authors reported underexpression of the HSP70-interacting protein ST13 and coexpression and coinduction of SNCA gene with critical enzymes of heme metabolism, in patients with PD versus healthy individuals. These findings suggest that blood and neuronal cells might have a common regulatory mechanism for gene expression. Additional cohort studies are warranted that would include larger PD population size, adequately selected on the basis of disease onset, date of medication initiation and follow-up program. In this study paradigm, PD patients could be followed during the course of the disease for correlation in their gene expression profile in blood to identify a PD biomarker panel phenotype that can potentially be used for diagnosis of early PD (risk markers) and monitor disease progression and its modification by therapy.

\section{Future perspective}

The rapid growth of molecular genetic, imaging and laboratory technology has expanded to the point at which the application of technically advanced BMs becomes broadly available and applicable. Such BMs and the additional occurrence of other BMs can be useful in mapping disease pathogenesis and identifying subjects at risk for $\mathrm{AD}$ and $\mathrm{PD}$.
Genetic BMs derived from genes mutated in familial PD/ $\mathrm{AD}$, or from transcriptomics of sporadic brain/blood tissue studies as well as polymorphisms in sporadic cases, may assist to identify individuals "at-risk" during the asymptomatic period, as well as to monitor disease progression. Early screening, detection, and diagnosis of AD and PD will allow intervention with disease modifying therapies at earlier stages and thus may potentially improve clinical outcome. Neurochemical BMs measured in the periphery by means of proteomics/metabolomics in blood plasma, CSF and other tissues may be useful adjuncts to imaging and clinical assessment tools for AD and PD. They may also provide valuable information about pathogenic mechanisms during clinical testing of neuroprotective/disease modifying drugs, which is especially relevant to personalised treatment.

In spite of the intensive research in the field of BMs for neurodegenerative diseases, there is some frustration with the progress being made. Many of the BMs discussed above show group differences (e.g. between normals and diseased), but none so far has been able to reliably diagnose $\mathrm{AD}$ or $\mathrm{PD}$ in their preclinical stages in an individual subject. It is possible that this depends on inaccurate diagnosis, since at present the diagnosis depends on clinical or pathological phenotypes, which suffer however from heterogeneity. This, added to the phenotypic convergence, indicate the limit of present-day understanding of these diseases. Since both AD and PD have a long preclinical period, future efforts should be focused on this time window to begin a neuroprotective treatment. Large multicenter studies are warranted to evaluate the diagnostic value of the combined application of BMs with multiple modalities for prediction, targeted prevention and evaluation of disease modifying therapies in $\mathrm{AD}$ and PD.

\section{References}

1. Weiner WJ. A differential diagnosis of Parkinsonism. Rev Neurol Dis. 2005;2:124-31.

2. Poewe W, Wenning G. The differential diagnosis of Parkinson's disease. Eur J Neurol. 2002;9 Suppl 3:23-30.

3. Galasko D, Hansen LA, Katzman R, et al. Clinicalneuropathological correlations in Alzheimer's disease and related dementias. Arch Neurol. 1994;51:888-95.

4. Hulette C, Nochlin D, McKeel D, et al. Clinical-neuropathologic findings in multi-infarct dementia: a report of six autopsied cases. Neurology. 1997;48:668-72.

5. Hulka BS, Wilcosky T. Biological markers in epidemiologic research. Arch Environ Health. 1988;43:83-9.

6. Naylor S. Biomarkers: current perspectives and future prospects. Expert Rev Mol Diagn. 2003;3:525-9.

7. Masterman DL, Mendez MF, Fairbanks LA, et al. Sensitivity, specificity, and positive predictive value of technetium 99HMPAO SPECT in discriminating Alzheimer's disease from other dementias. J Geriatr Psychiatry Neurol. 1997;10:15-21. 
8. Fratiglioni L, Launer LJ, Andersen K, et al. Incidence of dementia and major subtypes in Europe: a collaborative study of population-based cohorts. Neurologic Diseases in the Elderly Research Group. Neurology. 2000;54:S10-5.

9. Lobo A, Launer LJ, Fratiglioni L, et al. Prevalence of dementia and major subtypes in Europe: a collaborative study of population-based cohorts. Neurologic Diseases in the Elderly Research Group. Neurology. 2000;54:S4-9.

10. Graham JE, Rockwood K, Beattie BL, et al. Prevalence and severity of cognitive impairment with and without dementia in an elderly population. Lancet. 1997;349:1793-6.

11. Kawas C, Gray S, Brookmeyer R, et al. Age-specific incidence rates of Alzheimer's disease: the Baltimore Longitudinal Study of Aging. Neurology. 2000;54:2072-7.

12. McKhann G, Drachman D, Folstein M, et al. Clinical diagnosis of Alzheimer's disease: report of the NINCDS-ADRDA Work Group under the auspices of Department of Health and Human Services Task Force on Alzheimer's Disease. Neurology. 1984;34:939-44.

13. Lyketsos CG, Szekely CA, Mielke MM, et al. Developing new treatments for Alzheimer's disease: the who, what, when, and how of biomarker-guided therapies. Int Psychogeriatr. 2008;20: 871-89.

14. Sunderland T, Hampel H, Takeda M, et al. Biomarkers in the diagnosis of Alzheimer's disease: are we ready? J Geriatr Psychiatry Neurol. 2006;19:172-9.

15. Clark CM, Davatzikos C, Borthakur A, et al. Biomarkers for early detection of Alzheimer pathology. Neurosignals. 2008;16: 11-8.

16. Halperin I, Korczyn AD. Mild cognitive impairment (MCI): characteristics, risk factors and prevention. Harefuah. 2006;145:229-34.

17. Korczyn AD. Mixed dementia-the most common cause of dementia. Ann N Y Acad Sci. 2002;977:129-34.

18. Andreasen N, Minthon L, Davidsson P, et al. Evaluation of CSFtau and CSF-Abeta42 as diagnostic markers for Alzheimer disease in clinical practice. Arch Neurol. 2001;58:373-9.

19. Hanninen T, Hallikainen M, Tuomainen S, et al. Prevalence of mild cognitive impairment: a population-based study in elderly subjects. Acta Neurol Scand. 2002;106:148-54.

20. Jack Jr CR, Petersen RC, Xu Y, et al. Rates of hippocampal atrophy correlate with change in clinical status in aging and AD. Neurology. 2000;55:484-9.

21. Gasser T. Overview of the genetics of parkinsonism. Adv Neurol. 2003;91:143-52.

22. Bertram L, Tanzi RE. Thirty years of Alzheimer's disease genetics: the implications of systematic meta-analyses. Nat Rev Neurosci. 2008;9:768-78.

23. Mulder C, Scheltens P, Visser JJ, et al. Genetic and biochemical markers for Alzheimer's disease: recent developments. Ann Clin Biochem. 2000;37(Pt 5):593-607.

24. Strittmatter WJ, Weisgraber KH, Huang DY, et al. Binding of human apolipoprotein $\mathrm{E}$ to synthetic amyloid beta peptide: isoform-specific effects and implications for late-onset Alzheimer disease. Proc Natl Acad Sci U S A. 1993;90:8098-102.

25. Tanzi RE, Bertram L. Twenty years of the Alzheimer's disease amyloid hypothesis: a genetic perspective. Cell. 2005;120:545-55.

26. Roses AD, Lutz MW, Amrine-Madsen H, et al. A TOMM40 variable-length polymorphism predicts the age of late-onset Alzheimer's disease. Pharmacogenomics J. 2009. doi:10.1038/ tpj.2009.69

27. Grossman I, Lutz MW, Crenshaw D, et al. A.D. Alzheimer's disease: diagnostics, prognostics and the road to prevention. EPMA-J. 2010; doi:10.1007/s13167-010-0024-3

28. Reiman EM, Caselli RJ, Chen K, et al. Declining brain activity in cognitively normal apolipoprotein E epsilon 4 heterozygotes: a foundation for using positron emission tomography to efficiently test treatments to prevent Alzheimer's disease. Proc Natl Acad Sci U S A. 2001;98:3334-9.

29. Reiman EM, Chen K, Alexander GE, et al. Functional brain abnormalities in young adults at genetic risk for late-onset Alzheimer's dementia. Proc Natl Acad Sci U S A. 2004;101: 284-9.

30. Bookheimer SY, Strojwas MH, Cohen MS, et al. Patterns of brain activation in people at risk for Alzheimer's disease. N Engl J Med. 2000;343:450-6.

31. Lindsten K, Menendez-Benito V, Masucci MG, et al. A transgenic mouse model of the ubiquitin/proteasome system. Nat Biotechnol. 2003;21:897-902.

32. Morgan D, Diamond DM, Gottschall PE, et al. A beta peptide vaccination prevents memory loss in an animal model of Alzheimer's disease. Nature. 2000;408:982-5.

33. Schenk D, Barbour R, Dunn W, et al. Immunization with amyloid-beta attenuates Alzheimer-disease-like pathology in the PDAPP mouse. Nature. 1999;400:173-7.

34. Rockenstein E, Crews L, Masliah E. Transgenic animal models of neurodegenerative diseases and their application to treatment development. Adv Drug Deliv Rev. 2007;59:1093-102.

35. Korczyn AD. The amyloid cascade hypothesis. Alzheimers Dement. 2008;4:176-8.

36. Corder EH, Saunders AM, Strittmatter WJ, et al. Gene dose of apolipoprotein E type 4 allele and the risk of Alzheimer's disease in late onset families. Science. 1993;261:921-3.

37. Csernansky JG, Wang L, Joshi S, et al. Early DAT is distinguished from aging by high-dimensional mapping of the hippocampus. Dementia of the Alzheimer type. Neurology. 2000;55:1636-43.

38. Dickerson BC, Goncharova I, Sullivan MP, et al. MRI-derived entorhinal and hippocampal atrophy in incipient and very mild Alzheimer's disease. Neurobiol Aging. 2001;22:747-54.

39. Rosen AC, Prull MW, Gabrieli JD, et al. Differential associations between entorhinal and hippocampal volumes and memory performance in older adults. Behav Neurosci. 2003;117:1150 60.

40. Leung KK, Barnes J, Ridgway GR, et al. Automated crosssectional and longitudinal hippocampal volume measurement in mild cognitive impairment and Alzheimer's disease. Neuroimage. 2010;51(4):1345-59.

41. Reiber H, Peter JB. Cerebrospinal fluid analysis: disease-related data patterns and evaluation programs. J Neurol Sci. 2001;184: 101-22.

42. DeKosky ST, Ikonomovic MD, Styren SD, et al. Upregulation of choline acetyltransferase activity in hippocampus and frontal cortex of elderly subjects with mild cognitive impairment. Ann Neurol. 2002;51:145-55.

43. Davis KL, Mohs RC, Marin D, et al. Cholinergic markers in elderly patients with early signs of Alzheimer disease. JAMA. 1999;281:1401-6.

44. Resnick SM, Goldszal AF, Davatzikos C, et al. One-year age changes in MRI brain volumes in older adults. Cereb Cortex. 2000;10:464-72.

45. Sullivan EV, Pfefferbaum A, Adalsteinsson E, et al. Differential rates of regional brain change in callosal and ventricular size: a 4-year longitudinal MRI study of elderly men. Cereb Cortex. 2002; 12:438-45.

46. Chetelat G, Baron JC. Early diagnosis of Alzheimer's disease: contribution of structural neuroimaging. Neuroimage. 2003; 18:525-41.

47. Connor DM, Benveniste H, Dilmanian FA, et al. Computed tomography of amyloid plaques in a mouse model of Alzheimer's disease using diffraction enhanced imaging. Neuroimage. 2009;46:908-14. 
48. Borthakur A, Wheaton AJ, Gougoutas AJ, et al. In vivo measurement of T1rho dispersion in the human brain at 1.5 tesla. J Magn Reson Imaging. 2004;19:403-9.

49. Klunk WE, Panchalingam K, Moossy J, et al. N-acetyl-Laspartate and other amino acid metabolites in Alzheimer's disease brain: a preliminary proton nuclear magnetic resonance study. Neurology. 1992;42:1578-85.

50. Kwo-On-Yuen PF, Newmark RD, Budinger TF, et al. Brain $\mathrm{N}$-acetyl-L-aspartic acid in Alzheimer's disease: a proton magnetic resonance spectroscopy study. Brain Res. 1994;667: 167-74.

51. Jessen F, Block W, Traber F, et al. Proton MR spectroscopy detects a relative decrease of $\mathrm{N}$-acetylaspartate in the medial temporal lobe of patients with AD. Neurology. 2000;55:684-8.

52. Schuff N, Capizzano AA, Du AT, et al. Selective reduction of $\mathrm{N}$-acetylaspartate in medial temporal and parietal lobes in AD. Neurology. 2002;58:928-35.

53. Kantarci K, Jack Jr CR, Xu YC, et al. Regional metabolic patterns in mild cognitive impairment and Alzheimer's disease: a 1H MRS study. Neurology. 2000;55:210-7.

54. Valenzuela MJ, Sachdev PS, Wen W, et al. Dual voxel proton magnetic resonance spectroscopy in the healthy elderly: subcortical-frontal axonal $\mathrm{N}$-acetylaspartate levels are correlated with fluid cognitive abilities independent of structural brain changes. Neuroimage. 2000;12:747-56.

55. MacKay S, Ezekiel F, Di Sclafani V, et al. Alzheimer disease and subcortical ischemic vascular dementia: evaluation by combining MR imaging segmentation and H-1 MR spectroscopic imaging. Radiology. 1996;198:537-45.

56. Leinonen V, Alafuzoff I, Aalto S, et al. Assessment of \{beta\}Amyloid in a frontal cortical brain biopsy specimen and by positron emission tomography with carbon 11-labeled Pittsburgh compound B. Arch Neurol. 2008;65(10):1304-9.

57. Foster NL, Heidebrink JL, Clark CM, et al. FDG-PET improves accuracy in distinguishing frontotemporal dementia and Alzheimer's disease. Brain. 2007;130:2616-35.

58. Silverman DH, Small GW, Chang CY, et al. Positron emission tomography in evaluation of dementia: regional brain metabolism and long-term outcome. JAMA. 2001;286:2120-7.

59. Jagust W. Molecular neuroimaging in Alzheimer's disease. NeuroRx. 2004;1:206-12.

60. Johnson KA, Holman BL, Rosen TJ, et al. Iofetamine I 123 single photon emission computed tomography is accurate in the diagnosis of Alzheimer's disease. Arch Intern Med. 1990;150: $752-6$.

61. Eberling JL, Jagust WJ, Reed BR, et al. Reduced temporal lobe blood flow in Alzheimer's disease. Neurobiol Aging. 1992;13: 483-91.

62. Johnson KA, Jones K, Holman BL, et al. Preclinical prediction of Alzheimer's disease using SPECT. Neurology. 1998;50: $1563-71$.

63. Herholz K, Salmon E, Perani D, et al. Discrimination between Alzheimer dementia and controls by automated analysis of multicenter FDG PET. Neuroimage. 2002;17:302-16.

64. Archer HA, Edison P, Brooks DJ, et al. Amyloid load and cerebral atrophy in Alzheimer's disease: an 11C-PIB positron emission tomography study. Ann Neurol. 2006;60:145-7.

65. Engler H, Forsberg A, Almkvist O, et al. Two-year follow-up of amyloid deposition in patients with Alzheimer's disease. Brain. 2006;129:2856-66.

66. Mosconi L. Brain glucose metabolism in the early and specific diagnosis of Alzheimer's disease. FDG-PET studies in MCI and AD. Eur J Nucl Med Mol Imaging. 2005;32:486-510.

67. Kemppainen NM, Aalto S, Wilson IA, et al. Voxel-based analysis of PET amyloid ligand [11C]PIB uptake in Alzheimer disease. Neurology. 2006;67:1575-80.
68. Machulda MM, Ward HA, Borowski B, et al. Comparison of memory fMRI response among normal, MCI, and Alzheimer's patients. Neurology. 2003;61:500-6.

69. Jagust W, Thisted R, Devous Sr MD, et al. SPECT perfusion imaging in the diagnosis of Alzheimer's disease: a clinicalpathologic study. Neurology. 2001;56:950-6.

70. Kung MP, Hou C, Zhuang ZP, et al. Binding of two potential imaging agents targeting amyloid plaques in postmortem brain tissues of patients with Alzheimer's disease. Brain Res. 2004;1025:98-105.

71. Petersen RC, Aisen PS, Beckett LA, et al. Alzheimer's Disease Neuroimaging Initiative (ADNI): clinical characterization. Neurology. 2010;74:201-9.

72. Shoji M, Golde TE, Ghiso J, et al. Production of the Alzheimer amyloid beta protein by normal proteolytic processing. Science. 1992;258:126-9.

73. Blennow K. Cerebrospinal fluid protein biomarkers for Alzheimer's disease. NeuroRx. 2004;1:213-25.

74. Blennow K, Vanmechelen E, Hampel H. CSF total tau, Abeta42 and phosphorylated tau protein as biomarkers for Alzheimer's disease. Mol Neurobiol. 2001;24:87-97.

75. Clark CM, Xie S, Chittams J, et al. Cerebrospinal fluid tau and beta-amyloid: how well do these biomarkers reflect autopsyconfirmed dementia diagnoses? Arch Neurol. 2003;60:1696702.

76. Arai H, Nakagawa T, Kosaka Y, et al. Elevated cerebrospinal fluid tau protein level as a predictor of dementia in memoryimpaired patients. Alzheim Res. 1997;3:211-3.

77. Visser PJ, Verhey F, Knol DL, et al. Prevalence and prognostic value of CSF markers of Alzheimer's disease pathology in patients with subjective cognitive impairment or mild cognitive impairment in the DESCRIPA study: a prospective cohort study. Lancet Neurol. 2009;8:619-27.

78. Boban M, Grbic K, Mladinov M, et al. Cerebrospinal fluid markers in differential diagnosis of Alzheimer's disease and vascular dementia. Coll Antropol. 2008;32 Suppl 1:31-6.

79. Hampel H, Buerger K, Kohnken R, et al. Tracking of Alzheimer's disease progression with cerebrospinal fluid tau protein phosphorylated at threonine 231. Ann Neurol. 2001;49: 545-6.

80. Buerger K, Teipel SJ, Zinkowski R, et al. CSF tau protein phosphorylated at threonine 231 correlates with cognitive decline in MCI subjects. Neurology. 2002;59:627-9.

81. Hulstaert F, Blennow $\mathrm{K}$, Ivanoiu A, et al. Improved discrimination of AD patients using beta-amyloid(1-42) and tau levels in CSF. Neurology. 1999;52:1555-62.

82. Samuels SC, Silverman JM, Marin DB, et al. CSF beta-amyloid, cognition, and APOE genotype in Alzheimer's disease. Neurology. 1999;52:547-51.

83. Tapiola T, Pirttila T, Mikkonen M, et al. Three-year follow-up of cerebrospinal fluid tau, beta-amyloid 42 and 40 concentrations in Alzheimer's disease. Neurosci Lett. 2000;280:119 22.

84. Jensen M, Schroder J, Blomberg M, et al. Cerebrospinal fluid A beta42 is increased early in sporadic Alzheimer's disease and declines with disease progression. Ann Neurol. 1999;45:504-11.

85. Van Everbroeck B, Green AJ, Pals P, et al. Decreased levels of Amyloid-beta 1-42 in cerebrospinal fluid of Creutzfeldt-Jakob disease patients. J Alzheimers Dis. 1999;1:419-24.

86. Otto M, Esselmann H, Schulz-Shaeffer W, et al. Decreased betaamyloid1-42 in cerebrospinal fluid of patients with CreutzfeldtJakob disease. Neurology. 2000;54:1099-102.

87. Kanemaru K, Kameda N, Yamanouchi H. Decreased CSF amyloid beta42 and normal tau levels in dementia with Lewy bodies. Neurology. 2000;54:1875-6. 
88. Ringman JM, Younkin SG, Pratico D, et al. Biochemical markers in persons with preclinical familial Alzheimer disease. Neurology. 2008;71:85-92.

89. Crystal HA, Davies P. Toward a plasma marker for Alzheimer disease: some progress, but still a long way to go. Neurology. 2008;70:586-7.

90. Ray S, Britschgi M, Herbert C, et al. Classification and prediction of clinical Alzheimer's diagnosis based on plasma signaling proteins. Nat Med. 2007;13:1359-62.

91. Hye A, Lynham S, Thambisetty M, et al. Proteome-based plasma biomarkers for Alzheimer's disease. Brain. 2006;129: 3042-50.

92. Montine TJ, Beal MF, Cudkowicz ME, et al. Increased CSF F2isoprostane concentration in probable AD. Neurology. 1999; 52:562-5.

93. Irizarry MC, Hyman BT. Brain isoprostanes: a marker of lipid peroxidation and oxidative stress in AD. Neurology. 2003;61:436-7.

94. Rosner S, Giladi N, Orr-Urtreger A. Advances in the genetics of Parkinson's disease. Acta Pharmacol Sin. 2008;29:21-34.

95. Farrer MJ. Genetics of Parkinson disease: paradigm shifts and future prospects. Nat Rev Genet. 2006;7:306-18.

96. Greenamyre JT, Betarbet R, Sherer TB. The rotenone model of Parkinson's disease: genes, environment and mitochondria. Parkinsonism Relat Disord. 2003;9 Suppl 2:S59-64.

97. Obeso JA, Rodriguez-Oroz MC, Rodriguez M, et al. Pathophysiology of the basal ganglia in Parkinson's disease. Trends Neurosci. 2000;23:S8-19.

98. Fearnley JM, Lees AJ. Ageing and Parkinson's disease: substantia nigra regional selectivity. Brain. 1991;114:2283-301.

99. Schapira AH, Bezard E, Brotchie J, et al. Novel pharmacological targets for the treatment of Parkinson's disease. Nat Rev Drug Discov. 2006;5:845-54.

100. Langston JW. The Parkinson's complex: parkinsonism is just the tip of the iceberg. Ann Neurol. 2006;59:591-6.

101. Braak H, Del Tredici K, Rub U, et al. Staging of brain pathology related to sporadic Parkinson's disease. Neurobiol Aging. 2003;24:197-211.

102. Braak H, Muller CM, Rub U, et al. Pathology associated with sporadic Parkinson's disease-where does it end? J Neural Transm Suppl. 2006;70;89-97.

103. Bloch A, Probst A, Bissig H, et al. Alpha-synuclein pathology of the spinal and peripheral autonomic nervous system in neurologically unimpaired elderly subjects. Neuropathol Appl Neurobiol. 2006;32:284-95.

104. Fahn S, Elton R, Committee UD. Unified Parkinson's disease rating scale. In: Fahn S, Marsden C, Calne DB, Goldstein M, editors. Recent developments in Parkinson's disease. Florham Park: Macmillan Health Care Information; 1987. p. 153-63.

105. Hoehn MM, Yahr MD. Parkinsonism: onset, progression and mortality. Neurology. 1967;17:427-42.

106. Tolosa E, Wenning G, Poewe W. The diagnosis of Parkinson's disease. Lancet Neurol. 2006;5:75-86.

107. Vendette M, Gagnon JF, Decary A, et al. REM sleep behavior disorder predicts cognitive impairment in Parkinson disease without dementia. Neurology. 2007;69:1843-9.

108. Postuma RB, Gagnon JF, Vendette M, et al. REM sleep behaviour disorder in Parkinson's disease is associated with specific motor features. J Neurol Neurosurg Psychiatry. 2008; 79:1117-21

109. Burn DJ. Beyond the iron mask: towards better recognition and treatment of depression associated with Parkinson's disease. Mov Disord. 2002; 17:445-54.

110. Remy P, Doder M, Lees A, et al. Depression in Parkinson's disease: loss of dopamine and noradrenaline innervation in the limbic system. Brain. 2005;128:1314-22.
111. McKinnon JH, Demaerschalk BM, Caviness JN, et al. Sniffing out Parkinson disease: can olfactory testing differentiate parkinsonian disorders? Neurologist. 2007;13:382-5.

112. Hudry J, Thobois S, Broussolle E, et al. Evidence for deficiencies in perceptual and semantic olfactory processes in Parkinson's disease. Chem Senses. 2003;28:537-43.

113. Montgomery Jr EB, Koller WC, LaMantia TJ, et al. Early detection of probable idiopathic Parkinson's disease: I. Development of a diagnostic test battery. Mov Disord. 2000;15:46773.

114. Montgomery Jr EB, Lyons K, Koller WC. Early detection of probable idiopathic Parkinson's disease: II. A prospective application of a diagnostic test battery. Mov Disord. 2000; 15:474-8.

115. Troster AI. Neuropsychological characteristics of dementia with Lewy bodies and Parkinson's disease with dementia: differentiation, early detection, and implications for "mild cognitive impairment" and biomarkers. Neuropsychol Rev. 2008;18:10319.

116. Korczyn AD. Autonomic nervous system disturbances in Parkinson's disease. Adv Neurol. 1990;53:463-8.

117. Wolters E, Braak H. Parkinson's disease: premotor clinicopathological correlations. J Neural Transm Suppl. 2006;70;30919 .

118. Sommer C, Lauria G. Skin biopsy in the management of peripheral neuropathy. Lancet Neurol. 2007;6:632-42.

119. Rossi A, Giovenali P, Benvenuti M, et al. Skin biopsy: a new diagnostic tool for autonomic dysfunctions in Parkinson's disease? Lancet Neurol. 2007;6:848-9.

120. Leroux P-D (1880) Contribution à l'Étude des Causes de la Paralysie Agitante (in French). Thèse de Paris, Imprimeur de la Faculté de Médecine.

121. Gowers WR. A manual of diseases of the nervous system. London: Churchill; 1886-1888.

122. Wider C, Foroud T, Wszolek ZK. Clinical implications of gene discovery in Parkinson's disease and parkinsonism. Mov Disord. 2010;25 Suppl 1:S15-20.

123. Polymeropoulos MH, Lavedan C, Leroy E, et al. Mutation in the alpha-synuclein gene identified in families with Parkinson's disease. Science. 1997;276:2045-7.

124. Kruger R, Kuhn W, Muller T, et al. Ala30Pro mutation in the gene encoding alpha-synuclein in Parkinson's disease. Nat Genet. 1998;18:106-8.

125. Zarranz JJ, Alegre J, Gomez-Esteban JC, et al. The new mutation, E46K, of alpha-synuclein causes Parkinson and Lewy body dementia. Ann Neurol. 2004;55:164-73.

126. Singleton AB, Farrer M, Johnson J, et al. alpha-Synuclein locus triplication causes Parkinson's disease. Science. 2003; 302:841.

127. Ibanez P, Bonnet AM, Debarges B, et al. Causal relation between alpha-synuclein gene duplication and familial Parkinson's disease. Lancet. 2004;364:1169-71.

128. Chartier-Harlin MC, Kachergus J, Roumier C, et al. Alphasynuclein locus duplication as a cause of familial Parkinson's disease. Lancet. 2004;364:1167-9.

129. Leroy E, Boyer R, Auburger G, et al. The ubiquitin pathway in Parkinson's disease. Nature. 1998;395:451-2.

130. Lowe J, McDermott H, Landon M, et al. Ubiquitin carboxylterminal hydrolase (PGP 9.5) is selectively present in ubiquitinated inclusion bodies characteristic of human neurodegenerative diseases. J Pathol. 1990;161:153-60.

131. Aharon-Peretz J, Rosenbaum H, Gershoni-Baruch R. Mutations in the glucocerebrosidase gene and Parkinson's disease in Ashkenazi Jews. N Engl J Med. 2004;351:1972-7.

132. Ozelius LJ, Senthil G, Saunders-Pullman R, et al. LRRK2 G2019S as a cause of Parkinson's disease in Ashkenazi Jews. N Engl J Med. 2006;354:424-5. 
133. Kitada T, Asakawa S, Hattori N, et al. Mutations in the parkin gene cause autosomal recessive juvenile parkinsonism. Nature. 1998;392:605-8.

134. Valente EM, Abou-Sleiman PM, Caputo V, et al. Hereditary early-onset Parkinson's disease caused by mutations in PINK1. Science. 2004;304:1158-60.

135. Bonifati V, Rizzu P, van Baren MJ, et al. Mutations in the DJ-1 gene associated with autosomal recessive early-onset parkinsonism. Science. 2003;299:256-9.

136. Ramirez A, Heimbach A, Grundemann J, et al. Hereditary parkinsonism with dementia is caused by mutations in ATP13A2, encoding a lysosomal type 5 P-type ATPase. Nat Genet. 2006;38:1184-91.

137. Shimura H, Hattori N, Kubo S, et al. Familial Parkinson disease gene product, parkin, is a ubiquitin-protein ligase. Nat Genet. 2000;25:302-5.

138. Plun-Favreau H, Klupsch K, Moisoi N, et al. The mitochondrial protease HtrA2 is regulated by Parkinson's disease-associated kinase PINK1. Nat Cell Biol. 2007;9:1243-52.

139. Park J, Lee SB, Lee S, et al. Mitochondrial dysfunction in Drosophila PINK1 mutants is complemented by parkin. Nature. 2006;441:1157-61.

140. Brooks DJ. Technology insight: imaging neurodegeneration in Parkinson's disease. Nat Clin Pract Neurol. 2008;4:267-77.

141. Eckert T, Tang C, Eidelberg D. Assessment of the progression of Parkinson's disease: a metabolic network approach. Lancet Neurol. 2007;6:926-32.

142. Berg D. Biomarkers for the early detection of Parkinson's and Alzheimer's disease. Neurodegener Dis. 2008;5:133-6.

143. Zecca L, Youdim MB, Riederer P, et al. Iron, brain ageing and neurodegenerative disorders. Nat Rev Neurosci. 2004;5:863-73.

144. Gerlach M, Double KL, Youdim MB, et al. Potential sources of increased iron in the substantia nigra of parkinsonian patients. $\mathrm{J}$ Neural Transm Suppl. 2006;70;133-42.

145. Koeppen AH. The history of iron in the brain. J Neurol Sci. 1995;134(Suppl):1-9.

146. Hallgren B, Sourander P. The effect of age on the nonhemin iron in the human brain. J Neurochem. 1958;3(1):41-51.

147. Jellinger K, Paulus W, Grundke-Iqbal I, et al. Brain iron and ferritin in Parkinson's and Alzheimer's diseases. J Neural Transm Park Dis Dement Sect. 1990;2:327-40.

148. Sofic E, Paulus W, Jellinger K, et al. Selective increase of iron in substantia nigra zona compacta of parkinsonian brains. J Neurochem. 1991;56:978-82.

149. Zecca L, Shima T, Stroppolo A, et al. Interaction of neuromelanin and iron in substantia nigra and other areas of human brain. Neuroscience. 1996;73:407-15.

150. Berg D, Siefker C, Becker G. Echogenicity of the substantia nigra in Parkinson's disease and its relation to clinical findings. J Neurol. 2001;248:684-9.

151. Berg D, Roggendorf W, Schroder U, et al. Echogenicity of the substantia nigra: association with increased iron content and marker for susceptibility to nigrostriatal injury. Arch Neurol. 2002;59:999-1005.

152. Harrington MG, Merril CR. Two-dimensional electrophoresis and "ultrasensitive" silver staining of cerebrospinal fluid proteins in neurological diseases. Clin Chem. 1984;30:1933-7.

153. Nagai Y, Ueno S, Saeki Y, et al. Decrease of the D3 dopamine receptor mRNA expression in lymphocytes from patients with Parkinson's disease. Neurology. 1996;46:791-5.

154. Caronti B, Antonini G, Calderaro C, et al. Dopamine transporter immunoreactivity in peripheral blood lymphocytes in Parkinson's disease. J Neural Transm. 2001;108:803-7.

155. Parker Jr WD, Boyson SJ, Parks JK. Abnormalities of the electron transport chain in idiopathic Parkinson's disease. Ann Neurol. 1989;26:719-23.
156. Benecke R, Strumper P, Weiss H. Electron transfer complexes I and IV of platelets are abnormal in Parkinson's disease but normal in Parkinson-plus syndromes. Brain. 1993;116(Pt 6):1451-63.

157. Haas RH, Nasirian F, Nakano K, et al. Low platelet mitochondrial complex I and complex II/III activity in early untreated Parkinson's disease. Ann Neurol. 1995;37:714-22.

158. Migliore L, Petrozzi L, Lucetti C, et al. Oxidative damage and cytogenetic analysis in leukocytes of Parkinson's disease patients. Neurology. 2002;58:1809-15.

159. Harrington MG, Fonteh AN, Biringer RG, et al. Prostaglandin D synthase isoforms from cerebrospinal fluid vary with brain pathology. Dis Markers. 2006;22:73-81.

160. Davis JW, Grandinetti A, Waslien CI, et al. Observations on serum uric acid levels and the risk of idiopathic Parkinson's disease. Am J Epidemiol. 1996;144:480-4.

161. Weisskopf MG, O'Reilly E, Chen H, et al. Plasma urate and risk of Parkinson's disease. Am J Epidemiol. 2007;166:561-7.

162. Schwarzschild MA, Schwid SR, Marek K, et al. Serum urate as a predictor of clinical and radiographic progression in Parkinson disease. Arch Neurol. 2008;65:716-23.

163. Bogdanov M, Matson WR, Wang L, et al. Metabolomic profiling to develop blood biomarkers for Parkinson's disease. Brain. 2008;131:389-96.

164. Ascherio A, LeWitt PA, Xu K, et al. Urate as a predictor of the rate of clinical decline in Parkinson disease. Arch Neurol. 2009;66:1460-8.

165. Varani K, Vincenzi F, Tosi A, et al. A2A adenosine receptor overexpression and functionality, as well as TNF-alpha levels, correlate with motor symptoms in Parkinson's disease. Faseb J. 2010;24:587-98.

166. Morelli M, Di Paolo T, Wardas J, et al. Role of adenosine A2A receptors in parkinsonian motor impairment and 1-DOPAinduced motor complications. Prog Neurobiol. 2007;83:293309.

167. Goldknopf IL. Blood-based proteomics for personalized medicine: examples from neurodegenerative disease. Expert Rev Proteomics. 2008;5:1-8.

168. Sheta EA, Appel SH, Goldknopf IL. 2D gel blood serum biomarkers reveal differential clinical proteomics of the neurodegenerative diseases. Expert Rev Proteomics. 2006;3:45-62.

169. Goldknopf IL, Sheta EA, Bryson J, et al. Complement C3c and related protein biomarkers in amyotrophic lateral sclerosis and Parkinson's disease. Biochem Biophys Res Commun. 2006;342:1034-9.

170. Zhang J, Sokal I, Peskind ER, et al. CSF multianalyte profile distinguishes Alzheimer and Parkinson diseases. Am J Clin Pathol. 2008;129:526-9.

171. Mollenhauer B, Cullen V, Kahn I, et al. Direct quantification of CSF alpha-synuclein by ELISA and first cross-sectional study in patients with neurodegeneration. Exp Neurol. 2008;213:315-25.

172. El-Agnaf OM, Salem SA, Paleologou KE, et al. Detection of oligomeric forms of alpha-synuclein protein in human plasma as a potential biomarker for Parkinson's disease. FASEB J. 2006;20:419-25.

173. Duran R, Barrero FJ, Morales B, et al. Plasma alpha-synuclein in patients with Parkinson's disease with and without treatment. Mov Disord. 2010;25:489-93.

174. Barbour R, Kling K, Anderson JP, et al. Red blood cells are the major source of alpha-synuclein in blood. Neurodegener Dis. 2008;5:55-9.

175. Michell AW, Luheshi LM, Barker RA. Skin and platelet alphasynuclein as peripheral biomarkers of Parkinson's disease. Neurosci Lett. 2005;381:294-8.

176. Maita C, Tsuji S, Yabe I, et al. Secretion of DJ-1 into the serum of patients with Parkinson's disease. Neurosci Lett. 2008;431: $86-9$. 
177. Waragai M, Wei J, Fujita M, et al. Increased level of DJ-1 in the cerebrospinal fluids of sporadic Parkinson's disease. Biochem Biophys Res Commun. 2006;345:967-72.

178. Waragai M, Nakai M, Wei J, et al. Plasma levels of DJ-1 as a possible marker for progression of sporadic Parkinson's disease. Neurosci Lett. 2007;425:18-22.

179. Zetterberg H, Ruetschi U, Portelius E, et al. Clinical proteomics in neurodegenerative disorders. Acta Neurol Scand. 2008;118:111.

180. Mandel S, Amit T, Kalfon L, et al. Applying transcriptomic and proteomic knowledge to Parkinson's disease drug discovery. Expert Opin Drug Discov. 2007;2:1225-40.
181. Sullivan PF, Fan C, Perou CM. Evaluating the comparability of gene expression in blood and brain. Am J Med Genet B Neuropsychiatr Genet. 2006;141B:261-8.

182. Burczynski ME, Dorner AJ. Transcriptional profiling of peripheral blood cells in clinical pharmacogenomic studies. Pharmacogenomics. 2006;7:187-202.

183. Scherzer CR, Eklund AC, Morse LJ, et al. Molecular markers of early Parkinson's disease based on gene expression in blood. Proc Natl Acad Sci U S A. 2007;104:955-60.

184. Scherzer CR, Grass JA, Liao Z, et al. GATA transcription factors directly regulate the Parkinson's disease-linked gene alphasynuclein. Proc Natl Acad Sci U S A. 2008;105:10907-12. 